Superconvergence of Recovered Gradients of Discrete Time/Piecewise Linear Galerkin Approximations for Linear and Nonlinear Parabolic Problems

\author{
M.F. Wheeler \\ J.R. Whiteman
}

March 1992

TR92-07 



\title{
Superconvergence of Recovered Gradients of Discrete Time/Piecewise Linear Galerkin Approximations for Linear and Nonlinear Parabolic Problems
}

\author{
M.F. Wheeler \\ Department of Mathematical Sciences \\ Rice University, Houston, Texas, U.S.A. \\ J.R. Whiteman \\ BICOM, Institute of Computational Mathematics \\ Department of Mathematics and Statistics \\ Brunel University, Uxbridge, Middlesex, UK
}

\section{Acknowledgement}

Much of the work for this paper was done whilst the authors were visitors at the Center for Applied Mathematics at Purdue University, and whilst Whiteman was a Visiting Professor at the Department of Mathematics, Texas A\&M University. The work of Whiteman was also supported by the United States Army Research, Development and Standardization Group, London under Contract No. DAJA45-89-C-003. All this support is gratefully acknowledged. 


\begin{abstract}
Superconvergent error estimates in $\ell_{2}\left(H^{1}\right)$ and $\ell_{\infty}\left(H^{1}\right)$ norms are derived for recovered gradients of finite difference in time/piecewise linear Galerkin approximations in space for linear and quasi-nonlinear parabolic problems in two space dimensions. The analysis extends previous results for elliptic problems to the parabolic context, and covers problems in regions with non-smooth boundaries under certain assumptions on the regularity of the solutions.
\end{abstract}





\section{Introduction}

\section{$1.1 \quad$ Background}

This paper is concerned with the derivation of superconvergent error estimates for recovered gradients of finite difference in time/piecewise linear Galerkin approximations in space for linear and nonlinear parabolic problems in two space dimensions. For the linear and quasi-nonlinear problems the analysis covers the cases of variable coefficients and of regions with nonsmooth domains under certain assumptions on the regularity of the solutions.

Superconvergent recovered gradient error estimates for elliptic problems having solutions with low regularity due to Wheeler and Whiteman [12] and Goodsell and Whiteman [4], [5], [13]-[15], which developed the work of Levine [7], are here extended to parabolic problems with variable coefficients and, using numerical integration with either interpolation of coefficients or Gaussian quadrature, $\ell_{2}\left(H^{1}\right)$ results are produced. The analysis has been further

developed to produce similarly $\ell_{2}\left(H^{1}\right)$ and $\ell_{\infty}\left(H^{1}\right)$ superconvergent estimates for quasi-nonlinear parabolic problems.

The application of gradient recovery for time independent problems was discussed by Krizek and Neittaanmaki [6] and Wheeler and Whiteman [12]. 
Thomeé et al. [10] have considered the recovery of gradients for the heat equation in domains with smooth boundary, and in this context have proved $\ell_{\infty}\left(\ell_{\infty}\right)$ superconvergent estimates. The results here involving interpolation of coefficients extend the work of Douglas and Dupont [1] who obtained optimal convergence results in $\ell_{\infty}\left(L_{2}\right)$ for nonlinear parabolic problems.

\subsection{Superconvergence results for elliptic problems}

The results for the parabolic problems of later sections of this paper are based on superconvergent recovered gradient error estimates for two dimensional elliptic problems. These are reviewed briefly and the notation is defined.

Let $\Omega \subset \mathbb{R}^{2}$ be a simply connected open bounded domain with polygonal boundary $\partial \Omega$. The function $u(\mathbf{x})$ satisfies

$$
\begin{gathered}
-\nabla \cdot(a(\mathbf{x}) \nabla u(\mathbf{x}))=f(\mathbf{x}), \quad \mathbf{x} \in \Omega, \\
u(\mathbf{x})=0, \quad x \in \partial \Omega,
\end{gathered}
$$

where $a(\mathbf{x})$ and $f(\mathbf{x})$ are continuous and their second derivatives are locally in $L_{\infty}(\Omega)$ and $a(\mathbf{x}) \geq d_{0}>0, \mathbf{x} \in \bar{\Omega}$. A weak formulation of (1.1) is set up by multiplying both sides by $v \in H_{0}^{1}(\Omega)$ and integrating to obtain

$$
A(u, v)=(f, v) \quad \forall v \in H_{0}^{1}(\Omega),
$$


where

$$
A(u, v) \equiv \int_{\Omega} a \nabla u \cdot \nabla v, \quad u, v \in H_{0}^{1}(\Omega)
$$

and

$$
(f, v) \equiv \int_{\Omega} f v, \quad v \in H_{0}^{1}(\Omega) .
$$

For convenience and ease of explanation the variable coefficients in (1.1) have been taken as a scalar rather than as a positive definite matrix, whilst homogeneous Dirichlet boundary conditions have been used in (1.2). The matrix of coefficients and more general boundary conditions could also be treated.

Following [15], it is assumed that for the region $\Omega$ of problem (1.1)(1.2) there exist subdomains $\Omega_{0}, \Omega_{1}, \Omega_{2}$, such that $\Omega_{0} \subset \subset \Omega_{1} \subset \subset \Omega_{2} \subset \Omega$ satisfying the following conditions

Condition $1 . \Omega_{0}, \Omega_{1}, \Omega_{2}$ are rectangular and are partitioned by a uniform isosceles right triangular mesh in the following way: each subdomain is the union of a finite number of squares each of which is subdivided into two triangles $T_{k}$ by the diagonal of positive slope.

Condition 2. $\Omega_{2}$ is remote from singularities of the solutions $u$ of (1.3) at corners of $\partial \Omega$, so that $u \in W_{\infty}^{3}\left(\Omega_{2}\right)$, and the regularity of $f$ is assumed to be 
such as to guarantee this.

The part of the region $\bar{\Omega}-\bar{\Omega}_{2}$ is covered with a quasiuniform triangular mesh which is compatible with the regular mesh in $\Omega_{2}$. Let $h_{k}$ denote the diameter of the escribing circle of any element $T_{k}$ and define $h \equiv$ $\max _{\text {elements }} h_{k}$. A finite dimensional subspace $S^{h} \subset H_{0}^{1}(\Omega)$, consisting of continuous piecewise linear functions, is defined over the triangular mesh partition of $\Omega$. The Galerkin problem approximating (1.3) is that of finding

$$
u_{h} \in S^{h} \ni A\left(u_{h}, v_{h}\right)=\left(f, v_{h}\right) \quad \forall v_{h} \in S^{h} .
$$

In (1.6) it has been assumed that all integrations have been performed exactly. If, as is usual, the integrations are performed numerically, the resulting problem is that in which

$$
u_{h}^{*} \in S^{h} \ni A^{*}\left(u_{h}^{*}, v_{h}\right)=\left(f, v_{h}\right)^{*} \quad \forall v_{h} \in S^{h},
$$

where $A^{*}$ and $(\cdot, \cdot)^{*}$ indicate numerical integration.

We wish to use gradient recovery at all vertices (nodes) of triangles in $\bar{\Omega}_{0}$ and, as in [4], we define for nodes internal to $\Omega_{0}$ the recovered gradient of $v_{h} \in S^{h}$ to be

$$
H_{k}\left(v_{h}\right) \equiv \frac{1}{6} \sum_{i=1}^{6}\left(\nabla v_{h}\right)_{T_{k}^{i}}
$$


where $T_{k}^{i}, i=1,2, \ldots, 6$, are the six triangles surrounding the node. For nodes on the boundary $\partial \Omega_{0}$ we define suitable specific recovery schemes, see [13], and these together with (1.7) produce recovered gradients of $v_{h}$ at all nodes of $\bar{\Omega}_{0}$. To these we now fit respectively to each component of the recovered gradient a piecewise linear function $\left(\nabla v_{h}^{R}\right)_{x} \in S^{h}$ and $\left(\nabla v_{h}^{R}\right)_{y} \in S^{h}$. This construction ensures that

$$
\left|\nabla u-\nabla u_{h}^{* R}\right|_{0, \Omega_{0}} \leq C\left(\left|u_{I}-u_{h}\right|_{1, \Omega_{0}}+h^{3}|u|_{3, \Omega_{0}}\right)
$$

where $u_{I} \in S^{h}$ is the piecewise linear interpolant to $u$. The following result, which uses result (1.9), is due to Whiteman and Goodsell [15].

Theorem 1.1 Let $u(\mathbf{x}) \in H_{0}^{1}(\Omega) \cap H^{q}(\Omega) \cap H^{3}\left(\Omega_{2}\right), 1<q<2$, be the solution of the weak problem (1.3) and $u_{h}^{*} \in S^{h}$ be the solution of the Galerkin problem with numerical integration, (1.7). If $\nabla u_{h}^{*}$ is the recovered gradient function derived using (1.8) then

$$
\left|\nabla u-\nabla u_{h}^{* R}\right|_{0, \Omega_{0}} \leq C(\omega) h^{2(q-1)}\left(|u|_{q, \Omega}+|u|_{3, \Omega_{2}}\right)
$$

where $\omega \in C^{\infty}(\Omega) \cap C_{0}^{\infty}(\bar{\Omega})$ is a cut-off function such that $\omega(\mathbf{x})=1, \mathbf{x} \in \Omega_{0}$, $\omega(\mathbf{x})=0, \mathbf{x} \in\left(\Omega-\bar{\Omega}_{1}\right) \cup \partial \Omega_{1}$. 
Remark 1.1 Result (1.10) has assumed the regularity $u \in H^{q}(\Omega), 1<q<2$, for the weak solution, thinking of lower regularity as arising from the data and the geometry of problems (1.1)-(1.2). It could be that, in spite of the geometry of the problem, the solution is in fact smoother than the above so that $u \in H^{2}(\Omega)$. In this latter case the estimate (1.10) becomes

$$
\left|\nabla u-\nabla u_{h}^{* R}\right|_{0, \Omega_{0}} \leq C(\omega) h^{1+p}\left(|u|_{2, \Omega}+|u|_{3, \Omega_{2}}\right)
$$

where the auxiliary problem

$$
\left.\begin{array}{rcc}
-\nabla \cdot(a \nabla \phi)=\psi & \text { in } & \Omega \\
\phi=0 & \text { on } & \partial \Omega,
\end{array}\right\}
$$

$\psi \in L_{2}(\Omega)$, is such that $\phi \in H^{1+p}(\Omega), 0<p \leq 1$.

The above theorem, remark, and recovery of gradients will now be applied for the space derivatives of parabolic problems. 


\section{Discrete Time Galerkin Procedures with}

\section{Quadrature for Linear Parabolic Problems}

\subsection{Weak formulation, notation and numerical schemes}

In this section we consider a linear parabolic problem with nonconstant coefficients, involving the elliptic operator of Section 1. For this problem we

derive superconvergent estimates for recovered spacial derivatives of discrete time piecewise linear Galerkin approximations. These estimates will be extended to more general linear problems and in Section 3 to quasi-nonlinear parabolic problems.

The function $u(\mathbf{x}, t)$ satisfies the equation

$$
\frac{\partial u(\mathbf{x}, t)}{\partial t}-\nabla \cdot(a(\mathbf{x}, t) \nabla u(\mathbf{x}, t))=f(\mathbf{x}, t), \quad \mathbf{x} \in \Omega, \quad t \in J \equiv(O, T]
$$

together with the boundary condition

$$
u(\mathbf{x}, t)=0, \quad \mathbf{x} \in \partial \Omega,
$$

and the initial condition

$$
u(\mathbf{x}, 0)=u_{0}(\mathbf{x}), \quad \mathbf{x} \in \Omega
$$


where, as in Section $1, \Omega \subset \mathbb{R}^{2}$ is a simply connected polygonal domain with boundary $\partial \Omega$. The functions $a(\mathbf{x}, t), f(\mathbf{x}, t),(\mathbf{x}, t) \in \Omega \times \mathbb{R}$ are assumed to be such that $a(\mathbf{x}, t) \in L_{\infty}\left((0, T) ; W_{\infty}^{2}(\Omega)\right)$ and $f(\mathbf{x}, t)$ is positive and bounded. It is also assumed that $u(\mathbf{x}, t)$, the solution of $(2.1)-(2.3)$ is such that

$$
u(\mathrm{x}, t) \in L_{\infty}\left(H^{q}(\Omega)\right) \cap L_{\infty}\left(H_{0}^{1}(\Omega)\right) \cap H^{1}\left(H^{q}(\Omega)\right) \cap L_{2}\left(H^{3}\left(\Omega_{2}\right)\right)
$$

where $1<q \leq 2$.

For convenience we have assumed homogeneous Dirichlet boundary conditions. General Dirichlet, Neumann and mixed boundary conditions can be treated with only minor modifications in the arguments.

In approximating problem (2.1)-(2.3) we use a continuous piecewise linear Galerkin technique in the space dimensions, whilst the time discretisation is treated using implicit single step finite difference techniques of the " $\theta$ "-type, where $0 \leq \theta \leq \frac{1}{2}$. These time discretisation methods are chosen because they are most commonly used in practical computations for genuine engineering problems.

We first set up the weak form of (2.1) by multiplying both sides of the equation by a test function $v(\mathbf{x}) \in H_{0}^{1}(\Omega)$ and integrating by parts so that we have the weak form of (2.1)-(2.3) in which, for any $t \in J, u(\mathbf{x}, t) \in H_{0}^{1}(\Omega) \times J$ 
satisfies

$$
\left(u_{t}(\mathbf{x}, t), v(\mathbf{x})\right)+A(t ; u(\mathbf{x}, t), v(\mathbf{x}))=(f(\mathbf{x}, t), v(\mathbf{x})), \quad v(\mathbf{x}) \in H_{0}^{1}(\Omega)
$$

where $u_{t} \equiv \partial u / \partial t$ and, for $v(\mathbf{x}) \in H_{0}^{1}(\Omega)$,

$$
\begin{gathered}
A(t ; \phi, \psi) \equiv \int_{\Omega} a(\mathbf{x}, t) \nabla(\phi(\mathbf{x})) \cdot \nabla(\psi(\mathbf{x})) d \mathbf{x} \\
(f(., t), v) \equiv \int_{\Omega} f(\mathbf{x}, t) v(\mathbf{x}) d \mathbf{x} .
\end{gathered}
$$

As in Section 1 we again define $S^{h} \subset H_{0}^{1}(\Omega)$ to be the space of piecewise linear functions on $\Omega$. The continuous time Galerkin problem approximating (2.5) is that in which $u_{h}(\mathbf{x}, t) \in S^{h} \times J$ satisfies

$$
\left(\left(u_{h}\right)_{t}(., t), v_{h}\right)+A\left(t ; u_{h}, v_{h}\right)=\left(f(., t), v_{h}\right) \quad \forall v_{h} \in S^{h}
$$

where

$$
A\left(t ; u_{h}, v_{h}\right) \equiv \int_{\Omega} a(\mathbf{x}, t) \nabla u_{h}(\mathbf{x}, t) \cdot \nabla v_{h} d \mathbf{x}
$$

and

$$
\left(f(., t), v_{h}\right) \equiv \int_{\Omega} f(\mathbf{x}, t) v_{h}(\mathbf{x}) d \mathbf{x}
$$

The time interval $[0, T]$ is discretised by letting $\Delta t=T / N$, where $N$ is a positive integer, and setting $t^{n}=n \Delta t, n=0,1,2, \ldots, N$. The $\theta$-type finite 
difference replacements will be at time levels $t^{n, \theta}$ where

$$
t^{n, \theta} \equiv \theta t^{n}+(1-\theta) t^{n+1}, \quad 0 \leq \theta \leq \frac{1}{2}, \quad n=0,1, \ldots, N-1
$$

Similarly for any function $\phi(\mathbf{x}, t) \in L_{2}(\Omega) \times L_{\infty}(J)$ for $n=0,1, \ldots, N-1$, we define

$$
\phi^{n, \theta} \equiv \theta \phi^{n}+(1-\theta) \phi^{n+1}, \quad 0 \leq \theta \leq \frac{1}{2},
$$

and also for $u_{t}\left(\mathbf{x}, t^{n}\right)=u_{t}^{n}$ the backward difference replacement

$$
\delta_{t} u^{n}=\frac{u^{n+1}-u^{n}}{\Delta t} \equiv \frac{u\left(x, t^{n+1}\right)-u\left(x, t^{n}\right)}{\Delta t}
$$

We shall adopt the following standard norm notation. If $w(\mathbf{x}, t)$ defined on $[0, T] \times \Omega$ is sufficiently smooth and $1 \leq p \leq \infty$, then

$$
\|w\|_{L_{p}((0, T), H)}=\|F(t)\|_{L_{p}(0, T)}
$$

where

$$
F(t)=\|\omega\|_{H}(t)
$$

and $H$ is a normed linear space with \|\|$_{H}$. In general we shall take $H$ to be a Sobolev space $H^{q}(\Omega)$ for some positive $q$.

In addition we define

$$
\|w\|_{s, \infty, \Omega} \equiv\|w\|_{L_{\infty}\left((0, T), W_{\infty}(\Omega)\right)}
$$




$$
\|w\|_{\infty, \Omega} \equiv\|w\|_{L_{\infty}\left((0, T), L_{\infty}(\Omega)\right)}
$$

where

$$
\begin{aligned}
\|\cdot\|_{s, p, \Omega} & \equiv\|\cdot\|_{W_{p}^{s}(\Omega)} \\
= & \left(\sum_{|\alpha| \leq s}\left\|D^{\alpha} \cdot\right\|_{L_{p}(\Omega)}^{2}\right)^{\frac{1}{2}} .
\end{aligned}
$$

For $p=2$ we have that

$$
\|\cdot\|_{s} \equiv\|\cdot\|_{s, 2, \Omega}
$$

For the corresponding seminorms we use the notation $|\cdot|$ and the summation in $(2.16)$ is for $|\alpha|=s$.

Lemma 2.1 Let $V=H_{0}^{1}(\Omega)$ and $Q(\cdot)=Q(x, t, u(x, t))$ be a function that is bounded above and below by positive constants $a_{0}$ and $a_{1}$. Further assume that $Q$ is Lipschitz continuous as a function of $u$ and that $|G|$ and $|\nabla \cdot Q(u) G|$ with $G \equiv \frac{\partial Q}{\partial t}$ are bounded on $\bar{\Omega} \times[0, T]$. We assume the elliptic regularity given by (1.12). Let $W \in S^{h}$ satisfy

$$
(Q(u) \nabla(u-W), \nabla v)=0, \quad v \in S^{h}
$$

If $u$ and $\frac{\partial u}{\partial t}$ belong to $L^{2}\left(0, T ; H^{q}(\Omega)\right), 1 \leq q \leq 2$, then $\nu \equiv u-W$ satisfies 


$$
\begin{aligned}
& \|\nu\|_{L^{2}\left(0, T ; L^{2}(\Omega)\right)}+\left\|\frac{\partial \nu}{\partial t}\right\|_{L^{2}\left(0, T ; L^{2}(\Omega)\right)} \\
& \leq K h^{q-1+p}\left[\|u\|_{\left.L^{2}(0, T) ; H^{q}(\Omega)\right)}+\left\|\frac{\partial u}{\partial t}\right\|_{L^{2}\left(0, T ; H^{q}(\Omega)\right)}\right] .
\end{aligned}
$$

Proof. It is easy to show that there exists a constant $C$ such that

$$
\|\nu\|_{1, \Omega} \leq C h^{q-1}\|u\|_{q, \Omega}
$$

Moreover, if $\alpha \in V$ is such that

$$
(Q(u) \nabla \alpha, \nabla v)=0, \quad v \in S^{h}
$$

then it follows by the "Nitsche lift" (see [9]) and elliptic regularity that there exists a constant $C^{* *}$ such that

$$
\|\alpha\|_{0, \Omega} \leq C^{* *} h^{p}\|\alpha\|_{1, \Omega}
$$

To obtain estimates for $\frac{\partial \nu}{\partial t}$ we employ arguments used in [11] and [2].

If we differentiate (2.18) with respect to $t$ we see that

$$
\begin{aligned}
\left(Q(u) \nabla \frac{\partial \nu}{\partial t}, \nabla v\right) & \equiv\left(\frac{\partial u}{\partial t} \frac{\partial Q}{\partial u} \nabla \nu, \nabla v\right) \\
& \equiv(G \nabla \nu, \nabla v), \quad v \in S^{h}
\end{aligned}
$$


One can easily show by standard arguments that

$$
\left\|\frac{\partial \nu}{\partial t}\right\|_{1} \leq C h^{q-1}\left[\|u\|_{q, \Omega}+\left\|\frac{\partial u}{\partial t}\right\|_{q, \Omega}\right]
$$

Let $\beta \in V$ satisfy

$$
(Q(u) \nabla \beta, \nabla v)=(G \nabla \nu, \nabla v), \quad v \in V
$$

Then if $\alpha \equiv \frac{\partial \nu}{\partial t}-\beta$ we see from(2.21), (2.22) and (2.24) that

$$
\begin{aligned}
& \|\alpha\|_{0, \Omega} \leq C h^{p}\|\alpha\|_{1, \Omega} \\
& \quad \leq C h^{p+q-1}\left[\|u\|_{q, \Omega}+\left\|\frac{\partial u}{\partial t}\right\|_{q, \Omega}\right] .
\end{aligned}
$$

The last inequality follows by noting that

$$
\|\beta\|_{1, \Omega} \leq C\|\nu\|_{1, \Omega}
$$

To bound $\|\beta\|_{0, \Omega}$ we let $\Phi \in V$ be such that

$$
(Q(u) \nabla \Phi, \nabla v)=(\beta, v), \quad v \in V
$$


Then

$$
\begin{aligned}
\|\beta\|_{0, \Omega}^{2}= & (Q(u) \nabla \Phi, \nabla \beta) \\
= & (G \nabla \nu, \nabla \Phi) \\
= & \left(Q(u) \nabla \nu, Q^{-1}(u) G \nabla \Phi\right) \\
= & \left(Q(u) \nabla \nu, \nabla\left(Q^{-1}(u) G \Phi-\chi\right)\right) \\
& -\left(Q(u) \nabla \nu, \Phi \nabla\left(Q^{-1}(u) G\right)\right), \quad \chi \in S^{h} \\
= & \left(Q(u) \nabla \nu, \nabla\left(Q^{-1}(u) G \Phi-\chi\right)\right) \\
& +\left(\nu, \nabla \cdot Q(u) \Phi \nabla\left(Q^{-1}(u) G\right)\right), \quad \chi \in S^{h} .
\end{aligned}
$$

Thus,

$$
\begin{aligned}
\|\beta\|_{0, \Omega}^{2} & \leq C h^{p}\|\nu\|_{1, \Omega}\|\Phi\|_{1+p, \Omega}+C\|\nu\|_{0, \Omega}\|\Phi\|_{1+p, \Omega} \\
& \leq C h^{p+q-1}\|\beta\|_{0, \Omega}^{2} .
\end{aligned}
$$

and the result is immediate.

Returning now to the discretisation of the parabolic problem (2.5) and using the replacement (2.13) in (2.5) with $v=v_{h} \in S^{h}$, we have a $t=t^{n, \theta}$ such that

$$
\left(\delta_{t} u^{n}, v_{h}\right)+A^{n, \theta}\left(u^{n, \theta}, v_{h}\right)=\left(f\left(\mathbf{x}, t^{n, \theta}\right), v_{h}\right)+\left(E^{n, \theta}, v_{h}\right), \quad v_{h} \in S^{h}
$$


where

$$
A^{n, \theta}\left(u^{n, \theta}, v_{h}\right) \equiv \int_{\Omega} a\left(\mathbf{x}, t^{n, \theta}\right) \nabla u\left(\mathbf{x}, t^{n, \theta}\right) \cdot \nabla v_{h} d \mathbf{x}
$$

and $E^{n, \theta}$ is the remainder arising from the time discretisation, i.e.

$$
E^{n, \theta} \equiv \delta_{t} u^{n}-u_{t}\left(\cdot, t^{n, \theta}\right)
$$

We are finally able to define the approximating problem for $(2.1)-(2.3)$ as that in which the solution $u_{h}^{*}(\mathbf{x})$

$$
u_{h}^{*}:\left\{t^{0}, t^{1}, \ldots, t^{N}\right\} \longrightarrow S^{h}
$$

satisfies

$$
\left(\delta_{t} u_{h}^{* n}, v_{h}\right)+A^{* n, \theta}\left(u_{h}^{* n, \theta}, v_{h}\right)=\left(f\left(., t^{n, \theta}\right), v_{h}\right)^{*} \quad \forall v_{h} \in S^{h}
$$

where $A^{* n, \theta}(\cdot, \cdot)$ is the numerical approximation to $A^{n, \theta}(\cdot, \cdot)$ produced by using numerical integration, as discussed in Section 1, either by using a quadrature rule or by interpolating to the coefficients $a(\mathbf{x}, t)$. We similarly define $\left(f\left(\cdot, t^{n, \theta}\right), v_{h}\right)^{*}$ to be the numerical approximation to $\left(f\left(\cdot, t^{n, \theta}\right), v_{h}\right)$.

For every $t \in J$ we now define the elliptic projection $\tilde{u}_{h}(\mathbf{x}, t) \in S^{h} \times J$ of $u$ by

$$
A\left(t ; \tilde{u}_{h}(\cdot, t), v_{h}\right) \equiv A\left(t ; u(\cdot, t), v_{h}\right) \quad \forall v_{h} \in S^{h}
$$


Then $\tilde{u}_{h}^{n} \equiv \tilde{u}_{h}\left(\mathbf{x}, t^{n}\right)$ is, from $(2.26)$, given by

$$
\begin{aligned}
\left(\delta_{t} \tilde{u}_{h}^{n}, v_{h}\right)+A^{* n, \theta}\left(\tilde{u}_{h}^{n, \theta}, v_{h}\right) & \\
= & \left(\delta_{t}\left(\tilde{u}_{h}^{n}-u^{n}\right), v_{h}\right)+A^{* n, \theta}\left(\tilde{u}_{h}^{n, \theta}, v_{h}\right) \\
& \quad-A^{n, \theta}\left(\left(\tilde{u}_{h}\left(\cdot, t^{n, \theta}\right), v_{h}\right)+\left(f\left(\cdot, t^{n, \theta}\right), v_{h}\right)+\left(E^{n, \theta}, v_{h}\right) .\right.
\end{aligned}
$$

For convenience we now define

$$
\begin{aligned}
\eta^{n} & \equiv u_{h}^{* n}-\tilde{u}_{h}^{n} . \\
\xi^{n} & \equiv \tilde{u}_{h}^{n}-u^{n}
\end{aligned}
$$

Then subtracting (2.30) from (2.28) we obtain, using the above notation,

$$
\begin{aligned}
\left(\delta_{t} \eta^{n}, v_{h}\right)+A^{* n, \theta}\left(\eta^{n, \theta}, v_{h}\right) & \\
= & \left(f\left(\cdot, t^{n, \theta}\right), v_{h}\right)^{*}-\left(f\left(\cdot, t^{n, \theta}\right), v_{h}\right)-\left(E^{n, \theta}, v_{h}\right) \\
& \quad-\left(\delta_{t} \xi^{n}, v_{h}\right)-A^{* n, \theta}\left(\tilde{u}_{h}^{n, \theta}, v_{h}\right)+A^{n, \theta}\left(\tilde{u}_{h}\left(\cdot, t^{n, \theta}\right), v_{h}\right) \\
\equiv & T_{1}^{n, \theta}\left(v_{h}\right)-\left(E^{n, \theta}, v_{h}\right)-\left(\delta_{t} \xi^{n}, v_{h}\right)+T_{2}^{n, \theta}\left(v_{h}\right),
\end{aligned}
$$

where

$$
\begin{aligned}
& T_{1}^{n, \theta}\left(v_{h}\right) \equiv f\left(\cdot, t^{n, \theta}, v_{h}\right)^{*}-\left(f\left(\cdot, t^{n, \theta}\right), v_{h}\right) \\
& T_{2}^{n, \theta}\left(v_{h}\right) \equiv A^{n, \theta}\left(\tilde{u}_{h}\left(\cdot, t^{n, \theta}\right), v-A^{* n, \theta}\left(\tilde{u}_{h}^{n, \theta}, v_{h}\right) .\right.
\end{aligned}
$$


Setting $v^{h}=\eta^{n, \theta}$ in (2.33) we obtain

$\left(\delta_{t} \eta^{n}, \eta^{n, \theta}\right)+A^{* n, \theta}\left(\eta^{n, \theta}, \eta^{n, \theta}\right)=T_{1}^{n, \theta}\left(\eta^{n, \theta}\right)-\left(E^{n, \theta}, \eta^{n, \theta}\right)-\left(\delta_{t} \xi^{n}, \eta^{n, \theta}\right)+T_{2}^{n, \theta}\left(\eta^{n, \theta}\right)$

But

$$
\begin{aligned}
\left(\delta_{t} \eta^{n}, \eta^{n, \theta}\right) & =\left(\frac{\eta^{n+1}-\eta^{n}}{\Delta t}, \theta \eta^{n}+(1-\theta) \eta^{n+1}\right) \\
& =\frac{1}{\Delta t}\left\{(1-\theta)\left\|\eta^{n+1}\right\|_{0, \Omega}^{2}-\theta\left\|\eta^{n}\right\|_{0, \Omega}^{2}-(1-2 \theta)\left(\eta^{n}, \eta^{n+1}\right)\right\} \\
& \geq \frac{1}{\Delta t}\left\{\left\{(1-\theta)-\left(\frac{1-2 \theta}{2}\right)\right\}\left\|\eta^{n+1}\right\|_{0, \Omega}^{2}-\left\{\theta+\frac{1-2 \theta}{2}\right\}\left\|\eta^{n}\right\|_{0, \Omega}^{2}\right\}
\end{aligned}
$$

so that

$$
\left(\delta_{t} \eta^{n}, \eta^{n, \theta}\right) \geq \frac{1}{2 \Delta t}\left\{\left\|\eta^{n+1}\right\|_{0, \Omega}^{2}-\left\|\eta^{n}\right\|_{0, \Omega}^{2}\right\}
$$

and hence

$$
\begin{aligned}
& \frac{1}{2 \Delta t}\left\{\left\|\eta^{n+1}\right\|_{0, \Omega}^{2}-\left\|\eta^{n}\right\|_{0, \Omega}^{2}\right\}+A^{* n, \theta}\left(\eta^{n, \theta}, \eta^{n, \theta}\right) \\
& \quad \leq\left|T_{1}^{n, \theta}\left(\eta^{n, \theta}\right)\right|+\left|\left(E^{n, \theta}, \eta^{n, \theta}\right)\right|+\left|\left(\delta_{t} \xi^{n}, \eta^{n, \theta}\right)\right|+\left|T_{2}^{n, \theta}\left(\eta^{n, \theta}\right)\right| .
\end{aligned}
$$

For the term $\left|T_{1}^{n, \theta}\left(\eta^{n, \theta}\right)\right|$ in (2.34) we have that

$$
\begin{aligned}
\left|T_{1}^{n, \theta}\left(\eta^{n, \theta}\right)\right| & \equiv\left|\left(f\left(\cdot, t^{n, \theta}\right), \eta^{n, \theta}\right)^{*}-\left(f\left(\cdot, t^{n, \theta}\right), \eta^{n, \theta}\right)\right| \\
& \leq C h^{2}\left\|f\left(\cdot, t^{n, \theta}\right)\right\|_{2, \Omega}\left\|\eta^{n, \theta}\right\|_{0, \Omega} \\
& \leq C h^{2}\left\|f\left(\cdot, t^{n, \theta}\right)\right\|_{2, \Omega}\left\{\left\|\eta^{n}\right\|_{0, \Omega}+\left\|\eta^{n+1}\right\|_{0, \Omega}\right\}
\end{aligned}
$$


using approximation theory and Lemmas 3.7 and 3.8 of [12].

In considering the term $\left|\left(E^{n, \theta}, \eta^{n, \theta}\right)\right|$ in (2.34), we have that

$$
\begin{aligned}
\left|\left(E^{n, \theta}, \eta^{n, \theta}\right)\right| & \leq C\left\|E^{n, \theta}\right\|_{0, \Omega}\left\|\eta^{n, \theta}\right\|_{0, \Omega} \\
& \leq C\left\|E^{n, \theta}\right\|_{0, \Omega}\left\{\left\|\eta^{n}\right\|_{0, \Omega}+\left\|\eta^{n+1}\right\|_{0, \Omega}\right\} .
\end{aligned}
$$

Using Taylor series expansions, it can easily be verified that

$$
\begin{aligned}
& \left\|E^{n, \theta}\right\|_{0, \Omega} \leq C(\Delta t)^{\frac{1}{2}}\left\|u_{t t}\right\|_{L^{2}\left(\left(t^{n}, t^{n+1}\right) ; L^{2}(\Omega)\right)}, \quad 0 \leq \theta<\frac{1}{2}, \\
& \left\|E^{n, \theta}\right\|_{0, \Omega} \leq C(\Delta t)^{\frac{3}{2}}\left\|u_{t t t}\right\|_{L^{2}\left(\left(t^{n}, t^{n+1}\right) ; L^{2}(\Omega)\right)}, \quad \theta=\frac{1}{2} .
\end{aligned}
$$

Now consider the term $T_{2}^{n, \theta}\left(\eta^{n, \theta}\right)$ in (2.34). We have that

$$
\begin{aligned}
T_{2}^{n, \theta}\left(\eta^{n, \theta}\right) \equiv & A\left(t^{n, \theta} ; \tilde{u}_{h}\left(\cdot, t^{n, \theta}\right), \eta^{n, \theta}\right)-A^{* n, \theta}\left(\tilde{u}_{h}^{n, \theta}, \eta^{n, \theta}\right) \\
= & A\left(t^{n, \theta} ; \tilde{u}_{h}\left(\cdot, t^{n, \theta}\right), \eta^{n, \theta}\right)-A\left(t^{n, \theta} ; \tilde{u}_{h}^{n, \theta}, \eta^{n, \theta}\right) \\
& +A\left(t^{n, \theta} ; \tilde{u}_{h}^{n, \theta}, \eta^{n, \theta}\right)-A^{* n, \theta}\left(\tilde{u}_{h}^{n, \theta}, \eta^{n, \theta}\right) .
\end{aligned}
$$

Consider the second pair of terms

$$
\begin{aligned}
& A\left(t^{n, \theta} ; \tilde{u}_{h}^{n, \theta}, \eta^{n, \theta}\right)-A^{* n, \theta}\left(\tilde{u}_{h}^{n, \theta}, \eta^{n, \theta}\right) \\
& \quad \equiv \int_{\Omega}\left(a\left(\mathbf{x}, t^{n, \theta}\right)-a^{*}\left(\mathbf{x}, t^{n, \theta}\right)\right) \nabla \tilde{u}_{h}^{n, \theta} \cdot \nabla \eta^{n, \theta} d \mathbf{x} .
\end{aligned}
$$


For the case of interpolation where $a^{*}(\cdot, \cdot)=a_{I}(\cdot, \cdot)$ this gives

$$
\left|A\left(t^{n, \theta}, \tilde{u}_{h}^{n, \theta}, \eta^{n, \theta}\right)-A^{* n, \theta}\left(\tilde{u}_{h}^{n, \theta}, \eta^{n, \theta}\right)\right| \leq C h^{2}\|a\|_{2, \infty, \Omega}\left|\tilde{u}_{h}^{n, \theta}\right|_{1, \Omega}\left|\eta^{n, \theta}\right|_{1, \Omega}
$$

For the quadrature case

$$
\begin{aligned}
& \left|A\left(t^{n, \theta} ; \tilde{u}_{h}^{n, \theta}, \eta^{n, \theta}\right)-A^{* n, \theta}\left(\tilde{u}_{h}^{n, \theta}, \eta^{n, \theta}\right)\right| \\
& \quad=\left|\sum_{k} \int_{T_{k}}\left(a\left(\cdot, t^{n, \theta}\right)-a\left(G_{k}, t^{n, \theta}\right)\right) \nabla \tilde{u}_{h}^{n, \theta} \cdot \nabla \eta^{n, \theta} d \mathbf{x}\right| \\
& \leq C h^{2}\|a\|_{2, \infty, \Omega}\left|\tilde{u}_{h}^{n, \theta}\right|_{1, \Omega}\left|\eta^{n, \theta}\right|_{1, \Omega} .
\end{aligned}
$$

Now

$$
\begin{aligned}
\left|\tilde{u}_{h}^{n, \theta}\right|_{1, \Omega} & \leq\left|\tilde{u}_{h}^{n, \theta}-u^{n, \theta}\right|_{1, \Omega}+\left|u^{n, \theta}\right|_{1, \Omega} \\
& \leq C\left(\left|u^{n, \theta}\right|_{1, \Omega}+\left|\xi^{n, \theta}\right|_{1, \Omega}\right) .
\end{aligned}
$$

Thus from (2.38)-(2.41) for $t \in\left(t^{n}, t^{n+1}\right)$ we have

$$
\begin{aligned}
& \mid\left(A\left(t^{n, \theta} ; \tilde{u}_{h}^{n, \theta}, \eta^{n, \theta}\right)-A^{* n, \theta}\left(\tilde{u}_{h}^{n, \theta}, \eta^{n, \theta}\right) \mid\right. \\
& \quad \leq C h^{2}\|a\|_{2, \infty, \Omega}\left(\left|u^{n, \theta}\right|_{1, \Omega}+\left\|\xi^{n, \theta}\right\|_{1, \Omega}\right)\left(\left|\eta^{n}\right|_{1, \Omega}+\left|\eta^{n+1}\right|_{1, \Omega}\right) .
\end{aligned}
$$


For the first two terms in the right hand side of (2.38) we have

$$
\begin{aligned}
& A\left(t^{n, \theta} ; \tilde{u}_{h}\left(\cdot, t^{n, \theta}\right), \eta^{n, \theta}\right)-A\left(t^{n, \theta} ; \tilde{u}_{h}^{n, \theta}, \eta^{n, \theta}\right) \\
& \quad=\int_{\Omega} a\left(\mathbf{x}, t^{n, \theta}\right) \nabla\left(\tilde{u}_{h}\left(\cdot, t^{n, \theta}\right)-\tilde{u}_{h}^{n, \theta}\right) \cdot \nabla \eta^{n, \theta} d \mathbf{x} \\
& \quad=\int_{\Omega} a\left(\mathbf{x}, t^{n, \theta}\right) \nabla\left(\tilde{u}_{h}\left(\cdot, t^{n, \theta}\right)-\theta \tilde{u}_{h}^{n}-(1-\theta) \tilde{u}_{h}^{n+1}\right) \cdot \nabla \eta^{n, \theta} d \mathbf{x} \\
& \leq\|a\|_{\infty, \Omega}\left|\tilde{u}_{h}\left(\cdot, t^{n, \theta}\right)-\theta \tilde{u}_{h}^{n}-(1-\theta) \tilde{u}_{h}^{n+1}\right|_{1, \Omega}\left|\eta^{n, \theta}\right|_{1, \Omega} \\
& \quad \leq\left\{\begin{array}{l}
\|a\|_{\infty, \Omega}\left|\eta^{n, \theta}\right|_{1, \Omega} \times C \Delta t^{\frac{1}{2}}\left|\left(\tilde{u}_{h}\right)_{t}\right|_{L^{2}\left(\left(t^{n}, t^{n+1}\right) ; H^{1}(\Omega)\right)}, \quad 0 \leq \theta<\frac{1}{2}, \\
\|a\|_{\infty, \Omega}\left|\eta^{n, \theta}\right|_{1, \Omega} \times C \Delta t^{\frac{3}{2}}\left|\left(\tilde{u}_{h}\right)_{t t}\right|_{L^{2}\left(\left(t^{n}, t^{n+1}\right) ; H^{1}(\Omega)\right)}, \theta=\frac{1}{2} .
\end{array}\right.
\end{aligned}
$$

Estimates (2.42) and (2.43) together provide a bound for $\left|T_{2}^{n, \theta}\left(\eta^{n, \theta}\right)\right|$.

Consider next the term $\left(\delta_{t} \xi^{n}, \eta^{n, \theta}\right)$ in $(2.34)$; by Cauchy-Schwarz

$$
\left|\left(\delta_{t} \xi^{n}, \eta^{n, \theta}\right)\right| \leq\left\|\delta_{t} \xi^{n}\right\|_{0, \Omega}\left\|\eta^{n, \theta}\right\|_{0, \Omega} .
$$

But

$$
\begin{aligned}
\left\|\delta_{t} \xi^{n}\right\|_{0, \Omega}^{2} & =\int_{\Omega}\left[\frac{1}{\Delta t} \int_{t^{n}}^{t^{n+1}} \xi_{t} d t\right]^{2} d \mathbf{x} \\
& \leq \frac{1}{\Delta t}\left\|\xi_{t}\right\|_{\left.L^{2}\left(t^{n}, t^{n+1}\right) ; L^{2}(\Omega)\right)}^{2} .
\end{aligned}
$$

Now $\xi_{t}=\frac{\partial}{\partial t}\left(\tilde{u}_{h}^{n}-u^{n}\right)$ and from Lemma 2.1 


$$
\begin{aligned}
& \left|\left(\delta_{t} \xi^{n}, \eta^{n, \theta}\right)\right| \leq h^{q-1+p} C\left(\|a\|_{\infty, \Omega},\left\|a_{t}\right\|_{\infty, \Omega} \times\right. \\
& \quad\left(\|u\|_{L^{2}\left(\left(t^{n}, t^{n+1}\right) ; H^{q}(\Omega)\right)}+\left\|u_{t}\right\|_{L^{2}\left(\left(t^{n}, t^{n+1}\right) ; H^{q}(\Omega)\right)}\right) \\
& \quad \times\left(\left\|\eta^{n}\right\|_{0, \Omega}+\left\|\eta^{n+1}\right\|_{0, \Omega}\right) .
\end{aligned}
$$

From (2.34) and the estimates (2.35)-(2.44) we find that

$$
\begin{aligned}
\frac{1}{2 \Delta t}\{ & \left.\left\|\eta^{n+1}\right\|_{0, \Omega}^{2}-\left\|\eta^{n}\right\|_{0, \Omega}^{2}\right\}+A^{* n, \theta}\left(\eta^{n, \theta}, \eta^{n, \theta}\right) \\
\leq & \left\{C h^{2}\left\|f\left(\cdot, t^{n, \theta}\right)\right\|_{2, \Omega}\right. \\
& +h^{q-1+p} C\left(\left\|a_{t}\right\|_{\infty, \Omega}\|a\|_{\infty, \Omega}\right) \times \\
& \left(\|u\|_{L^{2}\left(\left(t^{n}, t^{n+1}\right) ; H^{q}(\Omega)\right)}+\left\|u_{t}\right\|_{L^{2}\left(\left(t^{n}, t^{n+1}\right) ; H^{q}(\Omega)\right)}\right) \\
& \left.+C \Delta t^{\beta-\frac{1}{2}}\left\|\frac{\partial^{\beta+1} u}{\partial t^{\beta+1}}\right\|_{L^{2}\left(\left(t^{n}, t^{n+1}\right) ; L^{2}(\Omega)\right)}\right\}\left(\left\|\eta^{n}\right\|_{0, \Omega}+\left\|\eta^{n+1}\right\|_{0, \Omega}\right)+ \\
& +\left\{C h^{2}\|a\|_{2, \infty, \Omega}\left(\left|u^{n, \theta}\right|_{1, \Omega}+\left|\xi^{n, \theta}\right|_{1, \Omega}\right)\right. \\
& \left.+C\|a\|_{\infty, \Omega}(\Delta t)^{\beta-\frac{1}{2}}\left\|\frac{\partial^{\beta} \tilde{u}_{h}}{\partial t^{\beta}}\right\|_{L^{2}\left(\left(t^{n}+t^{n+1}\right) ; H^{1}(\Omega)\right)}\right\}\left|\eta^{n, \theta}\right|_{1, \Omega}
\end{aligned}
$$

where $\beta=1$ if $0 \leq \theta<\frac{1}{2}$ and $\beta=2$ if $\theta=\frac{1}{2}$.

Let $a_{0} \equiv \inf _{(0, T) \times \Omega} a^{*}(\mathbf{x}, t)$ and $a_{1}=\sup _{(0, T) \times \Omega} a^{*}(\mathbf{x}, t)$. Then

$$
A^{* n, \theta}\left(\eta^{n, \theta}, \eta^{n, \theta}\right) \geq a_{0}\left\|\nabla \eta^{n, \theta}\right\|_{0, \Omega}^{2}
$$


Thus, using (2.46) and the inequality $a b \leq \frac{1}{2 \epsilon} a^{2}+\frac{\epsilon}{2} b^{2}$ with $\epsilon=\frac{1}{2}$ for terms involving $\left\|\eta^{n}\right\|_{0, \Omega}$ and $\left\|\eta^{n+1}\right\|_{0, \Omega}$ and $\epsilon=a_{0}$ for terms involving $\left|\eta^{n, \theta}\right|_{1, \Omega}$ we obtain from (2.45) that

$$
\begin{aligned}
& \frac{1}{2 \Delta t}\left\{\left\|\eta^{n+1}\right\|_{0, \Omega}^{2}-\left\|\eta^{n}\right\|_{0, \Omega}^{2}\right\}+a_{0}\left\|\nabla \eta^{n, \theta}\right\|_{0, \Omega}^{2} \\
& \leq C h^{4}\left\|f\left(\cdot, t^{n, \theta}\right)\right\|_{2, \Omega}^{2}+C\left(\|a\|_{\infty, \Omega},\left\|a_{t}\right\|_{\infty, \Omega}\right) h^{2(q-1+p)} \\
& \quad \times\left\{\|u\|_{\left(L^{2}\left(t^{n}, t^{n+1}\right) ; H^{q}(\Omega)\right)}^{2}+\left\|u_{t}\right\|_{\left(L^{2}\left(t^{n}, t^{n+1}\right) ; H^{q}(\Omega)\right)}^{2}\right\} \\
& +C(\Delta t)^{2 \beta-1}\left\|\frac{\partial^{\beta+1} u}{\partial t^{\beta+1}}\right\|_{L^{2}\left(\left(t^{n}, t^{n+1}\right) ; L^{2}(\Omega)\right)}^{2} \\
& +C\left(a_{0}\right)\|a\|_{\left.L^{\infty}(0, T) ; W_{\infty}^{2}(\Omega)\right)}^{2}\left(h^{4}\left|u^{n, \theta}\right|_{1, \Omega}^{2}\right. \\
& \left.+\Delta t^{2 \beta-1}\left\|\frac{\partial^{\beta} u}{\partial t^{\beta}}\right\|_{L^{2}\left(\left(t^{n}, t^{n+1}\right) ; H^{1}(\Omega)\right)}^{2}\right) \\
& +\frac{a_{0}}{2}\left|\eta^{n, \theta}\right|_{1, \Omega}^{2}+C\left(\left\|\eta^{n}\right\|_{0, \Omega}^{2}+\left\|\eta^{n+1}\right\|_{0, \Omega}^{2}\right)
\end{aligned}
$$

where in (2.47) we used the fact that

$$
\left|\tilde{u}_{h}^{n, \theta}\right|_{1, \Omega} \leq C\left(a_{0}, a_{1}\right)\left|u^{n, \theta}\right|_{1, \Omega}
$$

and

$$
\left\|\frac{\partial^{\beta} \tilde{u}_{h}}{\partial t^{\beta}}\right\|_{0, \Omega} \leq C\left(a_{0},\|a\|_{W_{\infty}^{2}(\Omega)}\right)\left\|\frac{\partial^{\beta} u}{\partial t^{\beta}}\right\|_{1, \Omega} .
$$

Multiplying (2.47) by $2 \Delta t$ and summing over $n$ for $n=0,1,2 \ldots, N-1$, we 
obtain the estimate

$$
\begin{aligned}
& \left\|\eta^{N}\right\|_{0, \Omega}^{2}-\left\|\eta^{0}\right\|_{0, \Omega}^{2}+2 a_{0} \sum_{n=0}^{N-1} \Delta t\left|\eta^{n, \theta}\right|_{1, \Omega}^{2} \\
& \leq \bar{C}_{1} h^{2(q-1+p)}\left\{\sum_{n=0}^{N-1} \Delta t\left\|f\left(\cdot, t^{n, \theta}\right)\right\|_{2, \Omega}^{2}\right. \\
& +C\left(\|a\|_{\infty, \Omega},\left\|a_{t}\right\|_{\infty, \Omega}\right)\|u\|_{\left(H^{1}(0, T) ; H^{q}(\Omega)\right)}^{2} \\
& \left.+\|a\|_{\left.L^{\infty}(0, T) ; W_{\infty}^{2}(\Omega)\right)}^{2}\|u\|_{\left(L^{2}(0, T) ; H^{1}(\Omega)\right)}^{2}\right\}+ \\
& +\bar{C}_{2}(\Delta t)^{2 \beta}\left\{\left\|\frac{\partial^{\beta+1} u}{\partial t^{\beta+1}}\right\|_{L^{2}(0, T) ; L^{2}(\Omega)}^{2}\right. \\
& \left.+\|a\|_{\left(L^{\infty}(0, T) ; W_{\infty}^{2}(\Omega)\right)}^{2}\left\|\frac{\partial^{\beta} u}{\partial t^{\beta}}\right\|_{\left(L^{2}(0, T) ; H^{1}(\Omega)\right)}^{2}\right\} \\
& +\bar{C}_{3}\left\{\sum_{n=1}^{N-1} \Delta t\left\|\eta^{n}\right\|_{0, \Omega}^{2}+\Delta t\left\|\eta^{N}\right\|_{0, \Omega}^{2}\right\}
\end{aligned}
$$

which can be written as

$$
\begin{aligned}
& (1-C \Delta t)\left\|\eta^{N}\right\|_{0, \Omega}^{2}+a_{0} \sum_{n=1}^{N-1} \Delta t\left|\eta^{n, \theta}\right|_{1, \Omega}^{2} \\
& \leq C_{1} h^{2(q-1+p)}+C_{2}(\Delta t)^{2 \beta}+C_{3} \sum_{n=1}^{N-1} \Delta t\left\|\eta^{n}\right\|_{0, \Omega}^{2}
\end{aligned}
$$

where the constants $C_{1}, C_{2}, C_{3}$ depend on various derivatives of $f, A$ and $u$ as in (2.37).

Application of Gronwall's Lemma (see [2] and [3]) to (2.49) yields the following result which we state as a theorem: 
Theorem 2.1 Let $u(\mathrm{x}, t) \in L_{\infty}\left(H^{q}(\Omega) \cap H_{0}^{1}(\Omega)\right) \cap H^{1}\left(H^{q}(\Omega)\right) \cap L_{2}\left(H^{3}\left(\Omega_{2}\right)\right)$, $1<q \leq 2$ be the solution of the weak form (2.5) of the parabolic problem (2.1)-(2.3) defined in $\Omega \times J$. Let $S^{h} \in H_{0}^{1}(\Omega)$ denote the spaces of piecewise linear functions defined on the triangular partition of $\Omega$ as in Section 1 and let $\tilde{u}_{h}(\mathbf{x}, t) \in S^{h}$ be the elliptic projection of $u(\mathbf{x}, t)$. If $\ddot{u}_{h}^{n}(\mathbf{x})$ is the solution of (2.28) at $t^{n}$, where $J \equiv(0, T), T=N \Delta t$ and $t^{n}=n \Delta t$, then

$$
\begin{aligned}
& (1-\Delta t)\left\|u_{h}^{N}-\tilde{u}_{h}^{N}\right\|_{0, \Omega}^{2}+a_{0} \sum_{n=0}^{N-1} \Delta t\left\|u_{h}^{n, \theta}-\tilde{u}_{h}^{n, \theta}\right\|_{1, \Omega}^{2} \\
& \leq C_{1} h^{2(q-1+p)}+C_{2}(\Delta t)^{2 \beta}
\end{aligned}
$$

where $C_{1}$ and $C_{2}$ are constants, $1<q \leq 2, \beta=1$ when $0 \leq \theta<\frac{1}{2}$ and $\beta=2$ when $\theta=\frac{1}{2}$. Here $0<p \leq 1$ is a parameter arising as in (1.12).

At any time level $t^{n}, n=1, \ldots, N$, we now apply the recovery procedure described in Section 1 to $\stackrel{*}{u}_{h}^{n}$ to produce the recovered gradient function $\nabla u_{h}^{* n R}$. Thus, combining the inequality (1.10) of Theorem 1.1 with the result of Theorem 2.1, we now have the main (superconvergence) result of this section.

Theorem 2.2 If $u(\mathbf{x}, t)$ and $\stackrel{*}{u}_{h}^{n}(\mathbf{x})$ are as in Theorem 2.1, and all the hypotheses of that theorem hold, and if $\nabla \tilde{u}_{h}^{n, R}$ is the recovered gradient function 
at the time level $n, n=1,2, \ldots, N$, then

$$
\left.\left\|\nabla u-\nabla \stackrel{*}{u}_{h}^{R}\right\|\right|_{\left(L^{2}\left(0, t^{N}\right) ; L^{2}\left(\Omega_{0}\right)\right)} ^{2}=O\left(h^{2(q-1+p)}\right)+(\Delta t)^{2 \beta}
$$

where $1<q \leq 2,0<p \leq 1$, and $\beta=1$ if $0<\theta<\frac{1}{2}$ and $\beta=2$ if $\theta=\frac{1}{2}$, where

$$
\left.\|\nabla w\|\right|_{\left(L^{2}\left(0, t^{N}\right) ; L^{2}\left(\Omega_{0}\right)\right)} ^{2} \equiv \sum_{n=0}^{N-1} \Delta t\left|\nabla u\left(t^{n}\right)\right|_{L^{2}\left(\Omega_{0}\right)}^{2} .
$$




\section{$3 \ell_{2}(0, T)$ Recovery of Gradients for}

\section{Nonlinear Parabolic Problems}

We now extend the previous analysis to nonlinear parabolic problems in which the function $u(\mathbf{x}, t)$ satisfies the equation

$$
\begin{gathered}
\frac{\partial u(\mathbf{x}, t)}{\partial t}-\nabla \cdot(a(\mathbf{x}, t, u(\mathbf{x}, t)) \nabla u(\mathbf{x}, t))=f(\mathbf{x}, t, u(\mathbf{x}, t)), \\
\mathbf{x} \in \Omega, \quad t \in J \equiv(0, T],
\end{gathered}
$$

together with the boundary condition

$$
u(\mathbf{x}, t)=0, \quad \mathbf{x} \in \partial \Omega
$$

and the initial condition

$$
u(\mathbf{x}, 0)=u_{0}(\mathbf{x}), \quad \mathbf{x} \in \Omega
$$

The same regularity conditions on $f$ as in Section 2.1 are taken, and we further assume that $f$ and $a$ are Lipschitz continuous with respect to $u$ with Lipschitz constant $K$, and that the regularity of $u(\mathbf{x}, t)$ is as in (2.4).

The weak formulation corresponding to $(2.5)$ is, for any $t \in J$, to find $u(\mathbf{x}, t) \in H_{0}^{1}(\Omega) \times J$ such that 


$$
\begin{aligned}
& \left(u_{t}(\mathbf{x}, t), v(\mathbf{x})\right)+A(t ; u(\mathbf{x}, t), v(\mathbf{x})) \\
& \quad=f(\mathbf{x}, t, u(\mathbf{x}, t), v(\mathbf{x})) \quad \forall v(\mathbf{x}) \in H_{0}^{1}(\Omega),
\end{aligned}
$$

where

$$
A(t ; \phi, \psi) \equiv \int_{\Omega} a(\mathbf{x}, t, u(\mathbf{x}, t)) \nabla \phi(\mathbf{x}) \cdot \nabla \psi(\mathbf{x}) d \mathbf{x}
$$

and

$$
(f(\cdot, t, u(\cdot, t)), v) \equiv \int_{\Omega} f(\mathbf{x}, t, u(\mathbf{x}, t)) v(\mathbf{x}) d \mathbf{x}
$$

Equation (2.26) now becomes

$$
\begin{aligned}
&\left(\delta_{t} u^{n}, v_{h}\right)+A\left(t^{n, \theta} ; u\left(\cdot, t^{n, \theta}\right), v_{h}\right)=\left(f\left(\mathbf{x}, t^{n, \theta}, u\left(\mathbf{x}, t^{n, \theta}\right), v_{h}\right)\right. \\
&+\left(E^{n, \theta}, v_{h}\right) \quad \forall v_{h} \in S^{h}
\end{aligned}
$$

whilst the Galerkin problem with quadrature, corresponding to $(2.28)$, is

$$
\left(\delta_{t} u_{h}^{* n}, v_{h}\right)+A^{* n, \theta}\left(u_{h}^{* n, \theta}, v_{h}\right)=\left(f\left(\cdot, t^{n, \theta}, E^{*} u_{h}^{* n, \theta}\right), v_{h}\right)^{*}
$$

where the $A^{* n, \theta}$ is now defined as

$$
A^{* n, \theta}\left(\phi^{n, \theta}, v_{h}\right) \equiv \int_{\Omega} a^{*}\left(\mathbf{x}, t^{n, \theta}, E^{x} \phi^{n, \theta}\right) \nabla \phi^{n, \theta} \cdot \nabla v_{h} d \mathbf{x}
$$


Here the use of the $*$ in $a^{*}$ and $(f, \cdot)^{*}$ has the same definition as in Section 2 and

$$
\begin{aligned}
E^{x} \phi^{n, \theta} & \equiv \phi^{n}, \quad 0 \leq \theta<\frac{1}{2} \\
& \equiv \frac{3}{2} \phi^{n}-\frac{1}{2} \phi^{n-1}, \quad \theta=\frac{1}{2} .
\end{aligned}
$$

In the analysis below we denote by $f_{I}$ the linear interpolate in $S^{h}$ of $f$. Equation (2.30) correspondingly becomes

$$
\begin{aligned}
\left(\delta_{t} \tilde{u}_{h}^{n}, v_{h}\right)+A^{* n, \theta}\left(\tilde{u}_{h}^{n, \theta}, v_{h}\right) \\
=\quad\left(\delta_{t}\left(\tilde{u}_{h}^{n}-u^{n}\right), v_{h}\right)+A^{* n, \theta}\left(\tilde{u}_{h}^{n, \theta}, v_{h}\right) \\
\quad-A\left(t^{n, \theta}, \tilde{u}_{h}\left(\cdot, t^{n, \theta}\right), v_{h}\right) \\
\quad+\left(f\left(\cdot, t^{n, \theta}, u\left(\mathbf{x}, t^{n, \theta}\right)\right), v_{h}\right)+\left(E^{n, \theta}, v_{h}\right) .
\end{aligned}
$$

Modifications to extend the result of Theorem 2.1 to the current nonlinear problem involve only the reestimation of $T_{1}^{n, \theta}$ and $T_{2}^{n, \theta}$ for the present case. In order to do this we proceed as follows. Following (2.24) we now have that

$$
\begin{aligned}
T_{1}^{n, \theta}\left(v_{h}\right)= & \left(f\left(\mathbf{x}, t^{n, \theta}, E^{x} u_{h}^{* n, \theta}\right), v_{h}\right)^{*} \\
& -\left(f\left(\mathbf{x}, t^{n, \theta}, u\left(x, t^{n, \theta}\right)\right), v_{h}\right) .
\end{aligned}
$$

Let $\left\{z_{j}(x)\right\}_{j=1}^{M}$ be a nodal basis for $S^{h}$ corresponding to the set of nodes $x_{i}$ 
of the partition of $\Omega$, and define

$$
f_{I}(x, t, u(x)) \equiv \sum_{j=1}^{M} f\left(x_{j}, t, u\left(x_{j}\right)\right) z_{j} .
$$

Set $V_{h}=\eta^{n, \theta}$. We first consider the case of interpolation of coefficients. Thus

$$
\begin{aligned}
\left|T_{1}^{n, \theta}\left(\eta^{n, \theta}\right)\right|= & \mid\left(\sum_{j}\left(f\left(\mathbf{x}_{j}, t^{n, \theta}, E^{x} u_{h}^{* n, \theta}\left(x_{j}\right)\right)-f\left(\mathbf{x}_{j}, t^{n, \theta}, u\left(x_{j}, t^{n, \theta}\right)\right)\right) z_{j}, \eta^{n, \theta}\right) \\
& \left.+\left(f_{I}\left(\mathbf{x}, t^{n, \theta}, u\left(\mathbf{x}, t^{n, \theta}\right)\right)-f\left(\mathbf{x}, t^{n, \theta}, u\left(\mathbf{x}, t^{n, \theta}\right)\right)\right), \eta^{n, \theta}\right) \mid \\
\leq & \left|\sum_{j} \frac{\partial f}{\partial u}\left(\mathbf{x}_{j}, t^{n, \theta}, \nu_{j}\right)\left(E^{x} u_{h}^{* n, \theta}\left(\mathbf{x}_{j}\right)-u\left(\mathbf{x}_{j}, t^{n, \theta}\right) z_{j}, \eta^{n, \theta}\right)\right| \\
& +C h^{2}\left\|f\left(t^{n, \theta}\right)\right\|_{2, \Omega}\left\|\eta^{n, \theta}\right\|_{0, \Omega}
\end{aligned}
$$

where $\nu_{j}$ lies between $E^{x} u_{h}^{* n, \theta}\left(\mathbf{x}_{j}\right)$ and $u\left(\mathbf{x}_{j}, t^{n, \theta}\right)$.

Now, assuming that $|\partial f / \partial u| \leq K$, we have that

$$
\begin{aligned}
& \left|\sum_{j} \frac{\partial f}{\partial u}\left(\mathbf{x}_{j}, t^{n, \theta}, \nu_{j}\right)\left(E^{x} u_{h}^{* n, \theta}\left(\mathbf{x}_{j}\right)-u\left(\mathbf{x}_{j}, t^{n, \theta}\right)\right) z_{j}\right| \\
\leq & K \sum_{j}\left|E^{x} u_{h}^{* n, \theta}\left(\mathbf{x}_{j}\right)-E^{x} u_{I}^{n, \theta}\left(\mathbf{x}_{j}\right)\right| z_{j} \\
& +\sum_{j}\left|E^{x} u_{I}^{n, \theta}\left(\mathbf{x}_{j}\right)-u_{I}\left(\mathbf{x}_{j}, t^{n, \theta}\right)\right| z_{j} .
\end{aligned}
$$

Thus, 


$$
\begin{aligned}
\left|T_{1}^{n, \theta}\left(\eta^{n, \theta}\right)\right| \leq & K\left[\left\|\sum_{j}\left|E^{x} u_{h}^{* n, \theta}\left(\mathbf{x}_{j}\right)-E^{x} u_{I}^{n, \theta}\left(\mathbf{x}_{j}\right)\right| z_{j}\right\|_{0, \Omega}\right. \\
& \left.+\left\|\sum_{j}\left|E^{x} u_{I}^{n, \theta}\left(\mathbf{x}_{j}\right)-u_{I}\left(\mathbf{x}_{j}, t^{n, \theta}\right)\right| z_{j}\right\|_{0, \Omega}\right]\left\|\eta^{n, \theta}\right\|_{0, \Omega} \\
& +C h^{2}\left\|f\left(t^{n, \theta}\right)\right\|_{2, \Omega}\left\|\eta^{n, \theta}\right\|_{0, \Omega} \\
\leq & C(K)\left[\left\|\left(u_{h}^{*}-u_{I}\right)^{n}\right\|_{0, \Omega}+\left\|\left(u_{h}^{*}-u_{I}\right)^{n-1}\right\|_{0, \Omega}+\right. \\
& \left.+(\Delta t)^{\beta}\left\|\frac{\partial^{\beta} u_{I}^{n, \theta}}{\partial t^{\beta}}\right\| \|_{0, \Omega}\right]\left\|\eta^{n, \theta}\right\|_{0, \Omega} \\
& +C h^{2}\left\|f\left(t^{n, \theta}\right)\right\|_{2, \Omega}\left\|\eta^{n, \theta}\right\|_{0, \Omega}
\end{aligned}
$$

where $\beta=1,0 \leq \theta<\frac{1}{2}$ and $\beta=2, \theta=\frac{1}{2}$.

Here we have used the equivalence of norms for finite dimensional spaces, i.e.

$$
\left\|\sum\left|\alpha_{j}\right| z_{j}\right\|_{0, \Omega} \approx\left\|\sum \alpha_{j} z_{j}\right\|_{0, \Omega}
$$

We simplify the bound (3.14) as follows: 


$$
\begin{aligned}
& \left|T_{1}^{n, \theta}\left(\eta^{n, \theta}\right)\right| \leq C(K)\left\{\left\|\eta^{n}\right\|_{0, \Omega}^{2}+\left\|\eta^{n-1}\right\|_{0, \Omega}^{2}\right. \\
& +\left\|\xi^{n}\right\|_{0, \Omega}^{2}+\left\|\xi^{n-1}\right\|_{0, \Omega}^{2}+\left\|\left(u_{I}-u\right)^{n}\right\|_{0, \Omega}^{2} \\
& +\left\|\left(u_{I}-u\right)^{n-1}\right\|_{0, \Omega}^{2}+(\Delta t)^{2 \beta}\left\|\frac{\partial^{\beta} u}{\partial t^{\beta}}\left(\bar{t}^{n}\right)\right\|_{0, \Omega}^{2} \\
& \left.+\left\|\eta^{n, \theta}\right\|_{0, \Omega}^{2}\right\}+C h^{4}\left\|f\left(t^{n, \theta}\right)\right\|_{2, \Omega} \\
& \leq C(K)\left\{\left\|\eta^{n}\right\|_{0, \Omega}^{2}+\left\|\eta^{n-1}\right\|_{0, \Omega}^{2}\right. \\
& \left.+h^{2(q-1+p)} \sum_{\ell=n-1}^{n}\left\|u^{\ell}\right\|_{2, \Omega}^{2}+(\Delta t)^{2 \beta}\left\|\frac{\partial^{\beta} u}{\partial t^{\beta}}\left(\bar{t}^{n}\right)\right\|_{1, \Omega}^{2}\right\} \\
& +C h^{4}\left\|f\left(t^{n, \theta}\right)\right\|_{2, \Omega}+C\left\|\eta^{n+1}\right\|_{0, \Omega}^{2}
\end{aligned}
$$

where $\bar{t}^{n}$ is a point between $t^{n-1}$ and $t^{n, \theta}$.

Now using Gauss quadrature we have

$$
\begin{aligned}
T_{1}^{n, \theta}\left(\eta^{n, \theta}\right) & =\sum_{k} \int_{T_{k}}\left[f\left(G_{k}, t^{n, \theta}, E^{x} u_{h}^{* n, \theta}\left(G_{k}\right)\right)-f\left(\mathbf{x}, t^{n, \theta}, u\left(\mathbf{x}, t^{n, \theta}\right)\right)\right] \eta^{n, \theta} d \mathbf{x} \\
& =\sum_{k} \int_{T_{k}}\left[S_{1 k}^{n, \theta}+S_{2 k}^{n, \theta}+S_{3 k}^{n, \theta}+S_{\mathbf{4} k}^{n, \theta}\right] \eta^{n, \theta} d x
\end{aligned}
$$

where

$$
\begin{aligned}
S_{1 k}^{n, \theta} & \equiv f\left(G_{k}, t^{n, \theta}, E^{x} u_{h}^{m, \theta}\left(G_{k}\right)\right)-f\left(G_{k}, t^{n, \theta}, E^{x} u_{I}^{n, \theta}\left(G_{k}\right)\right) \\
S_{2 k}^{n, \theta} & \equiv f\left(G_{k}, t^{n, \theta}, E^{x} u_{I}^{n, \theta}\left(G_{k}\right)\right)-f\left(\mathbf{x}, t^{n, \theta}, E^{x} u_{I}^{n, \theta}(x)\right) \\
S_{3 k}^{n, \theta} & \equiv f\left(\mathbf{x}, t^{n, \theta}, E^{x} u_{I}^{n, \theta}(x)\right)-f\left(x, t^{n, \theta}, u_{I}\left(x, t^{n, \theta}\right)\right)
\end{aligned}
$$




$$
S_{4 k}^{n, \theta} \equiv f\left(\mathbf{x}, t^{n, \theta}, u_{I}\left(x, t^{n, \theta}\right)\right)-f\left(x, t^{n, \theta}, u\left(x, t^{n, \theta}\right)\right)
$$

Using the Lipschitz continuity of $f$ and the definition of $E^{x}$, we have that

$$
\begin{aligned}
& \sum_{k} \int_{T_{k}}\left(S_{3 k}^{n, \theta}+S_{4 k}^{n, \theta}\right) \eta^{n, \theta} d \mathbf{x} \\
& \leq C\left[(\Delta t)^{2 \beta-1}\left\|\frac{\partial^{\beta} u}{\partial t^{\beta}}\right\|_{L^{2}\left(\left(t^{n}, t^{n+1}\right), L^{2}(\Omega)\right)}^{2}+K\left\|u-u_{I}\right\|_{L_{\infty}\left((0, T), L^{2}(\Omega)\right)}^{2}\right] \\
& \quad+C\left\|\eta^{n, \theta}\right\|_{0, \Omega}^{2}
\end{aligned}
$$

Now for simplicity we consider first $S_{2 k}^{n, \theta}$.

$$
\begin{aligned}
\sum_{k} \int_{T_{k}} S_{2 k}^{n, \theta} \eta^{n, \theta} d x & \equiv \sum_{k} \int_{T_{k}} S_{2 k}^{n, \theta}\left(\eta^{n, \theta}-\eta^{n, \theta}\left(G_{k}\right)\right) \\
& \leq \sum_{k} h_{k}^{2}\left\|f\left(\mathbf{x}, t^{n, \theta}, E^{x} u_{I}^{n, \theta}(\mathbf{x})\right)\right\|_{1, T_{k}}\left\|\eta^{n, \theta}\right\|_{1, T k} \\
& \leq a_{0}\left\|\eta^{n, \theta}\right\|_{1, \Omega}^{2}+C h^{4}\|f\|_{1, \Omega}^{2}
\end{aligned}
$$

In obtaining (3.22) we have used the fact that for any continuous $F(\mathbf{x})$

$$
\int_{T_{k}}\left(F(\mathbf{x})-F\left(G_{k}\right)\right) \cdot 1 d \mathbf{x}=0
$$

thus,

$$
\int_{T_{k}}\left(F(\mathbf{x})-F\left(G_{k}\right)\right) \theta(\mathbf{x}) d x=\int_{T_{k}}\left(F(\mathbf{x})-F\left(G_{k}\right)\right)(\theta(\mathbf{x})-\text { const }) d \mathbf{x}
$$

Now turning to $S_{1 k}^{n, \theta}$, we have that 


$$
\begin{aligned}
\sum_{k} \int_{T_{k}} S_{1 k}^{n, \theta} d \mathbf{x} & \\
= & \sum_{k}\left\{\int _ { T _ { k } } \left[f\left(G_{k}, t^{n, \theta}, E^{x} u_{h}^{* n, \theta}\left(G_{k}\right)\right)-f\left(G_{k}, t^{n, \theta}, E^{x} u_{I}^{n, \theta}\left(G_{k}\right)\right)\right.\right. \\
& \left.\quad-f\left(\mathbf{x}, t^{n, \theta}, E^{x} u_{h}^{* n, \theta}(\mathbf{x})\right)+f\left(\mathbf{x}, t^{n, \theta}, E^{x} u_{I}(\mathbf{x})\right)\right] \eta^{n, \theta} \\
\quad & \quad \int_{T_{k}}\left[f\left(\mathbf{x}, t^{n, \theta}, E^{x} u_{h}^{* n, \theta}(\mathbf{x})\right)-f\left(\mathbf{x}, t^{n, \theta}, E^{x} u_{I}(\mathbf{x})\right)\right] \eta^{n, \theta} d \mathbf{x}
\end{aligned}
$$

By applying the mean value theorem and Gauss quadrature estimates to (3.23) we have that

$$
\begin{aligned}
\sum_{k} \int_{T_{k}} S_{1 k}^{n, \theta} d x \leq & \sum_{k} h_{k}\left\|\eta^{n, \theta}\right\|_{1, T_{k}}\left|f\left(\cdot, t^{n, \theta}, E^{x} u_{h}^{* n, \theta}(\cdot)\right)-f\left(\cdot, t^{n, \theta}, E^{x} u_{I}(\cdot)\right)\right|_{0, T_{k}}+ \\
& +K\left\|E^{x} u_{h}^{* n, \theta}-E^{x} u_{I}^{n, \theta}\right\|_{0, \Omega}\left\|\eta^{n, \theta}\right\|_{0, \Omega} \\
\leq & \sum_{k}\left\|\eta^{n, \theta}\right\|_{1, T_{k}} h_{k}\left\|\frac{\partial f}{\partial u}\right\|_{L_{\infty}\left((0, T) ; L_{\infty}(\Omega)\right)}\left\|E^{x} u_{I}^{n, \theta}-E^{x} u_{h}^{* n, \theta}\right\|_{0, T_{k}} \\
& +K\left\|E^{x} u_{h}^{* n, \theta}-E^{x} u_{I}^{n, \theta}\right\|_{0, \Omega}\left\|\eta^{n, \theta}\right\|_{0, \Omega}
\end{aligned}
$$

Thus

$$
\begin{aligned}
\sum_{k} \int_{T_{k}} S_{1 k}^{n, \theta} d \mathbf{x} \leq & h^{2}\left\|\eta^{n, \theta}\right\|_{1, \Omega}^{2}+C\left\|\eta^{n+1}\right\|_{0, \Omega}^{2} \\
& +C\left(\left\|\eta^{n}\right\|_{0, \Omega}^{2}+\left\|\eta^{n-1}\right\|_{0, \Omega}^{2}\right) \\
& +C \sum_{\ell=n-1}^{n}\left\|\left(\tilde{u}_{h}-u_{I}\right)^{\ell}\right\|_{0, \Omega}^{2}
\end{aligned}
$$


Combining (3.21), (3.22) and(3.24) we have an estimate for $T_{1}^{n, \theta}\left(\eta^{n, \theta}\right)$. The only terms which did not appear (for the linear problem) in (2.45) are the $\left\|\eta^{n-1}\right\|_{0, \Omega}^{2}$ and $\left\|\left(\tilde{u}_{h}-u_{I}\right)^{\ell}\right\|_{0, \Omega}^{2}, \ell=n-1, n$, in $(3.24)$. These of course arise from the extrapolation which was not needed in the linear case. This has no effect on the final estimate, as in (2.45), because of the application of the Gronwall inequality and approximation theory.

We now seek to estimate the term $T_{2}^{n, \theta}\left(\eta^{n, \theta}\right)$, as in $(2.27)$, for the nonlinear context. The term $T_{2}^{n, \theta}\left(\eta^{n, \theta}\right)$ is defined as

$$
\begin{aligned}
T_{2}^{n, \theta}\left(\eta^{n, \theta}\right)= & -A^{* n, \theta}\left(\tilde{u}_{h}^{n, \theta}, \eta^{n, \theta}\right) \\
& +A\left(t^{n, \theta} ; \tilde{u}_{h}\left(\cdot, t^{n, \theta}\right), \eta^{n, \theta}\right) .
\end{aligned}
$$

In a similar manner to the treatment of $T_{1}^{n, \theta}\left(\eta^{n, \theta}\right)$ for the case of the interpolation of coefficients we have that

$$
\begin{aligned}
T_{2}^{n, \theta}\left(\eta^{n, \theta}\right)= & -\int_{\Omega} \sum_{j} a\left(\mathbf{x}_{j}, t^{n, \theta}, E^{x} u_{h}^{* n, \theta}\left(\mathbf{x}_{j}\right)\right) z_{j} \nabla \tilde{u}_{h}^{n, \theta} \cdot \nabla \eta^{n, \theta} d \mathbf{x} \\
& +\int_{\Omega} a\left(\mathbf{x}, t^{n, \theta}, u\left(\mathbf{x}, t^{n, \theta}\right)\right) \nabla \tilde{u}_{h}\left(\cdot, t^{n, \theta}\right) \cdot \nabla \eta^{n, \theta} d \mathbf{x} \\
= & \int_{\Omega} \sum_{j}\left(-a\left(\mathbf{x}_{j}, t^{n, \theta}, E^{x} u_{h}^{* n, \theta}\left(\mathbf{x}_{j}\right)\right)+a\left(\mathbf{x}_{j}, t^{n, \theta}, E^{x} u_{I}^{n, \theta}\left(\mathbf{x}_{j}\right)\right) z_{j} \nabla \tilde{u}_{h}^{n, \theta} \cdot \nabla \eta^{n, \theta} d \mathbf{x}\right. \\
& +\int_{\Omega} \sum_{j}-a\left(\mathbf{x}_{j}, t^{n, \theta}, E^{x} u_{I}^{n, \theta}\left(\mathbf{x}_{j}\right)+a\left(\mathbf{x}_{j}, t^{n, \theta}, u_{I}\left(\mathbf{x}_{j}, t^{n, \theta}\right)\right) z_{j} \nabla \tilde{u}_{h}^{n, \theta} \cdot \nabla \eta^{n, \theta} d \mathbf{x}\right. \\
& +\int_{\Omega}\left(\sum_{j}-a\left(\mathbf{x}_{j}, t^{n, \theta}, u_{I}\left(\mathbf{x}_{j}, t^{n, \theta}\right)\right) z_{j}+a\left(\mathbf{x}, t^{n, \theta}, u\left(\mathbf{x}, t^{n, \theta}\right)\right)\right) \nabla \tilde{u}_{h}^{n, \theta} \cdot \nabla \eta^{n, \theta} d \mathbf{x}
\end{aligned}
$$




$$
+\int a\left(\mathbf{x}, t^{n, \theta}, u\left(\mathbf{x}, t^{n, \theta}\right)\left(-\nabla \tilde{u}_{h}^{n, \theta}+\nabla \tilde{u}_{h}\left(\cdot, t^{n, \theta}\right)\right) \cdot \nabla \eta^{n, \theta} d \mathbf{x}\right.
$$

The proof proceeds similarly to the treatment of the $T_{1}^{n, \theta}\left(\eta^{n, \theta}\right)$ terms. In particular

$$
\begin{aligned}
& \left|T_{2}^{n, \theta}\left(\eta^{n, \theta}\right)\right| \\
& \leq \quad K\left[\left\|\sum_{j}\left|E^{x} u_{h}^{* n, \theta}\left(\mathbf{x}_{j}\right)-E^{x} u_{I}^{n, \theta}\left(\mathbf{x}_{j}\right)\right| z_{j}\right\|_{0, \Omega}\right. \\
& \quad+\left\|\sum_{j}\left|E^{x} u_{I}^{n, \theta}\left(\mathbf{x}_{j}\right)-u_{I}\left(\mathbf{x}_{j}, t^{n, \theta}\right)\right| z_{j}\right\|_{0, \Omega} \\
& \left.\quad+C h^{2}\|a\|_{2, \Omega}\left(t^{n, \theta}\right)\right]\left\|\nabla \tilde{u}_{h}\right\|_{\infty, \Omega}\left\|\nabla \eta^{n, \theta}\right\|_{0, \Omega} \\
& \quad+C(\Delta t)^{\beta-\frac{1}{2}}\left\|\frac{\partial^{\beta} u}{\partial t^{\beta}}\right\|_{L^{2}\left(\left(t^{n}, t^{n+1}\right) ; H^{1}(\Omega)\right)}\left\|\nabla \eta^{n, \theta}\right\|_{0, \Omega}
\end{aligned}
$$

so that

$$
\begin{aligned}
\left|T_{2}^{n, \theta}\left(\eta^{n, \theta}\right)\right| \leq & C\left(K,\|\nabla \tilde{u}\|_{\infty, \Omega}\right) \times \\
& \left\{\left\|\eta^{n}\right\|_{0, \Omega}^{2}+\left\|\eta^{n-1}\right\|_{0, \Omega}^{2}+h^{2(q-1+p)} \sum_{\ell=n-1}^{n}\left\|u^{\ell}\right\|_{2, \Omega}^{2}\right. \\
& \left.+(\Delta t)^{2 \beta-1}\left\|\frac{\partial^{\beta} u}{\partial t^{\beta}}\right\|_{L^{2}\left(\left(t^{n}, t^{n+1}\right) ; H^{1}(\Omega)\right)}^{2}+h^{4}\|a\|_{2, \Omega}^{2}\left(t^{n, \theta}\right)\right\} \\
& +\frac{a_{0}}{4}\left\|\eta^{n, \theta}\right\|_{0, \Omega}^{2} .
\end{aligned}
$$


We remark that for $q=2$

$$
\begin{aligned}
& \left\|\nabla \tilde{u}_{h}\right\|_{\infty, \Omega} \\
& \leq \quad\|\nabla u\|_{\infty, \Omega}+h^{-1}\left\|\nabla\left(u_{I}-\tilde{u}_{h}\right)\right\|_{L^{\infty}(0, T) ; L^{2}(\Omega)} \\
& \quad+\left\|\nabla\left(u-u_{I}\right)\right\|_{\infty, \Omega} \\
& =0(1)
\end{aligned}
$$

since

$$
\left\|\nabla\left(u_{I}-\tilde{u}_{h}\right)\right\|_{L^{\infty}\left((0, T) ; L^{2}(\Omega)\right)} \leq h^{q-1}
$$

In the case of Gauss quadrature the treatment of $T_{2}^{n, \theta}\left(\eta^{n, \theta}\right)$ simplifies since $\nabla \tilde{u}_{h}^{n, \theta} \cdot \nabla \eta^{n, \theta}$ is constant over $T_{k}$. More precisely, we have

$$
\begin{aligned}
T_{2}^{n, \theta}\left(\eta^{n, \theta}\right) \equiv & \sum_{k}\left(-\int_{T_{k}} a\left(G_{k}, t^{n, \theta}, E^{x} u_{h}^{* n, \theta}\left(G_{k}\right)\right) \nabla \tilde{u}_{h}^{n, \theta} \cdot \nabla \eta^{n, \theta} d \mathbf{x}\right. \\
& \left.+\int_{T_{k}} a\left(\mathbf{x}, t^{n, \theta}, u\left(\mathbf{x}, t^{n, \theta}\right)\right) \nabla \tilde{u}_{h}^{n, \theta} \cdot \nabla \eta^{n, \theta} d \mathbf{x}\right) \\
= & \sum_{k}\left(-\int_{T_{k}} a\left(\mathbf{x}, t^{n, \theta}, E^{x} u_{h}^{* n, \theta}(\mathbf{x})\right) \nabla \tilde{u}_{h}^{n, \theta} \cdot \nabla \eta^{n, \theta} d \mathbf{x}\right. \\
& \left.+\int_{T_{k}} a\left(\mathbf{x}, t^{n, \theta}, u\left(\mathbf{x}, t^{n, \theta}\right)\right) \nabla \tilde{u}_{h}^{n, \theta} \cdot \nabla \eta^{n, \theta} d x\right) \\
\leq & K\left\|E^{x} u_{h}^{* n, \theta}-u\left(\cdot, t^{n, \theta}\right)\right\|_{0, \Omega}\left\|\nabla \tilde{u}_{h}^{n, \theta}\right\|_{\infty, \Omega}\left\|\nabla \eta^{n, \theta}\right\|_{0, \Omega}
\end{aligned}
$$

The bounds for $T_{1}^{n, \theta}\left(\eta^{n, \theta}\right)$ and $T_{2}^{n, \theta}\left(\eta^{n, \theta}\right)$ include no new terms over those for the linear case except the $\left\|\eta^{n-1}\right\|_{0, \Omega}$ and $\sum_{\ell=n-1}^{n}\left\|\left(\tilde{u}_{h}-u_{I}\right)^{\ell}\right\|_{0, \Omega}^{2}$ so that 
an estimate of the type (2.47) can be obtained and this leads immediately to the theorem

Theorem 3.1 Let $u(\mathbf{x}, t)$ be the solution of (3.1)-(3.3) and have the same regularity as that given by (2.4). We further assume with $a(t)=a(t, u(t))$ the elliptic regularity assumption for $q=2$. Let $u_{h}^{* n}(\mathbf{x})$ be defined by (3.8) with $u_{h}^{* 0}=\tilde{u}_{h}^{0}$ or $u_{I}\left(\cdot, t^{0}\right)$. Then

$$
\begin{gathered}
(1-\Delta t)\left\|\eta^{N}\right\|_{0, \Omega}^{2}+a_{0} \sum_{n=1}^{N-1} \Delta t\left\|\eta^{n, \theta}\right\|_{1, \Omega}^{2} \\
\leq C_{1} h^{2(q-1+p)}+C_{1}(\Delta t)^{2 \beta}
\end{gathered}
$$

where $\beta=1$ when $0 \leq \theta<\frac{1}{2}$ and $\beta=2$ when $\theta=\frac{1}{2}$.

In addition the following theorem results immediately from Theorem 3.1 , and the recovery results of Section 2 .

Theorem 3.2 Let $u(\mathbf{x}, t)$ and $u_{h}^{*}(\mathbf{x}, t)$ satisfy the hypotheses of Theorem 3.1 and let $\nabla u_{h}^{* n R}$ be the recovered gradient function at the time level $n, n=$ $1,2, \ldots, N$. Then (2.51) holds for $\left(\nabla u-\nabla u_{h}^{* R}\right)$ in the case of the nonlinear problem (3.1)-(3.3). 


\section{$4 \ell_{\infty}(0, T)$ Recovery of Gradients for}

\section{Nonlinear Parabolic Problems}

All the estimates derived so far in this paper have been in terms of the $L_{2}$ norm in time. From a practical point of view it is much more useful to have estimates which in time are in terms of the $\ell_{\infty}$-norm. We now derive an $\ell_{\infty}$ norm time estimate for the backward difference procedure $(\theta=0)$ as applied to the nonlinear problem (3.1)-(3.3). Whilst for simplicity we consider the case $\theta=0$, our arguments can be generalized in a straightforward fashion to include the case $0 \leq \theta \leq \frac{1}{2}$.

We proceed as in the previous sections and taking $\theta=0$ subtract equation (3.11) involving the elliptic projection $\tilde{u}_{h}$ from equation (3.8) involving the calculated Galerkin approximation $u_{h}^{*}$. For ease of notation in allowing variability of the third variable in $a(\cdot, \cdot, \cdot)$ we redefine the $A^{*}$ and $A$ to contain three arguments. Thus

$$
\begin{aligned}
& A^{* n+1}(z, \phi, \psi) \equiv \int_{\Omega} a^{*}\left(\mathbf{x}, t^{n+1}, z\right) \nabla \phi \cdot \nabla \psi d \mathbf{x} \\
& \equiv\left\{\begin{array}{l}
\int_{\Omega} a_{I}\left(\mathbf{x}, t^{n+1}, z\right) \nabla \phi \cdot \nabla \psi d \mathbf{x} \\
\sum_{k} \int_{T_{k}} a\left(G_{k}, t^{n+1}, z\left(G_{k}\right)\right) \nabla \phi \cdot \nabla \psi d \mathbf{x}
\end{array}\right.
\end{aligned}
$$


depending on whether interpolation or Gauss quadrature is used, and

$$
A(t ; z, \phi, \psi) \equiv \int_{\Omega} a(\mathbf{x}, t, z(\mathbf{x}, t)) \nabla \phi \cdot \nabla \psi d \mathbf{x}
$$

so that

$$
\begin{aligned}
\left(\delta_{t} \eta^{n}, v_{h}\right) & +A^{* n+1}\left(u_{h}^{* n}, \eta^{n+1}, v_{h}\right) \\
= & -A^{* n+1}\left(u_{h}^{* n}, \tilde{u}_{h}^{n+1}, v_{h}\right)+A^{* n+1}\left(u^{n}, \tilde{u}_{h}^{n+1}, v_{h}\right) \\
& -\left(\delta_{t} \xi^{n}, v_{h}\right)-A^{* n+1}\left(u^{n}, \tilde{u}_{h}^{n+1}, v_{h}\right)+A\left(t^{n+1} ; u^{n+1}, \tilde{u}_{h}^{n+1}, v_{h}\right) \\
& +\left(f\left(\mathbf{x}, t^{n+1}, u_{h}^{* n+1}\right), v_{h}\right)^{*}-\left(f\left(\mathbf{x}, t^{n+1}, u^{n+1}\right), v_{h}\right)-\left(E^{n, \theta} v_{h}\right)
\end{aligned}
$$

Setting $v_{h}=\delta_{t} \eta^{n}$ in (4.3), multiplying the result by $\Delta t$ and summing the result over $n, n=0,1,2, \ldots, N-1$, we obtain

$$
\begin{aligned}
\sum_{n=0}^{N-1} \Delta t & \left\|\delta_{t} \eta^{n}\right\|_{0, \Omega}^{2}+\sum_{n=0}^{N-1} \Delta t A^{* n+1}\left(u_{h}^{* n}, \eta^{n+1}, \delta_{t} \eta^{n}\right) \\
= & \sum_{n=0}^{N-1} \Delta t\left(\tilde{T}_{1}^{n}+\tilde{T}_{2}^{n}+\tilde{T}_{3}^{n}\right) \\
& -\sum_{n=0}^{N-1} \Delta t\left[\left(\delta_{t} \xi^{n}, \delta_{t} \eta^{n}\right)+\left(E^{n, \theta}, \delta_{t} \eta^{n}\right)\right]
\end{aligned}
$$

where

$$
\begin{aligned}
& \tilde{T}_{1}^{n} \equiv-A^{* n+1}\left(u_{h}^{* n}, \tilde{u}_{h}^{n+1}, \delta_{t} \eta^{n}\right)+A^{* n+1}\left(u^{n}, \tilde{u}_{h}^{n+1}, \delta_{t} \eta^{n}\right) \\
& \tilde{T}_{2}^{n} \equiv-A^{* n+1}\left(u_{h}^{n}, \tilde{u}_{h}^{n+1}, \delta_{t} \eta^{n}\right)+A\left(t^{n+1} ; u^{n+1}, \tilde{u}_{h}^{n+1}, \delta_{t} \eta^{n}\right) \\
& \tilde{T}_{3}^{n} \equiv\left(f\left(\mathbf{x}, t^{n+1}, u_{h}^{* n+1}\right), \delta_{t} \eta^{n}\right)^{*}-\left(f\left(\mathbf{x}, t^{n+1}, u^{n+1}\right), \delta_{t} \eta^{n}\right) .
\end{aligned}
$$


Writing the time difference $\delta_{t} \eta^{n}$ as $\left(\eta^{n+1}-\eta^{n}\right) / \Delta t$ and taking $\eta^{0} \equiv 0$, the second term on the left hand side of (4.4) becomes

$$
\begin{aligned}
& \sum_{n=0}^{N-1} \Delta t A^{* n+1}\left(u_{h}^{* n}, \eta^{n+1}, \delta_{t} \eta^{n}\right) \\
& =\frac{1}{\Delta t} \sum_{n=0}^{N-1} \Delta t\left[A^{* n+1}\left(u_{h}^{* n}, \eta^{n+1}, \eta^{n+1}\right)-A^{* n+1}\left(u_{h}^{* n}, \eta^{n}, \eta^{n}\right)\right] \\
& \equiv \frac{1}{\Delta t}\left\{\sum_{n=1}^{N} \Delta t A^{* n}\left(u_{h}^{* n-1}, \eta^{n}, \eta^{n}\right)-\sum_{n=1}^{N-1} \Delta t A^{* n+1}\left(u_{h}^{* n}, \eta^{n}, \eta^{n}\right)\right\} \\
& \equiv A^{* N}\left(u_{h}^{* N-1}, \eta^{N}, \eta^{N}\right) \\
& \quad+\frac{1}{\Delta t}\left\{\sum_{n=1}^{N-1} \Delta t\left(A^{* n}\left(u_{h}^{* n-1}, \eta^{n}, \eta^{n}\right)-A^{* n+1}\left(u_{h}^{* n}, \eta^{n}, \eta^{n}\right)\right)\right\} \\
& \equiv A^{* N}\left(u_{h}^{* N-1}, \eta^{N}, \eta^{N}\right)-\sum_{n=0}^{N-1} \Delta t \tilde{T}_{4}^{n},
\end{aligned}
$$

where

$$
\begin{aligned}
& \tilde{T}_{4}^{n} \equiv \frac{-1}{\Delta t} \int_{\Omega}\left(a^{*}\left(\cdot, t^{n}, u_{h}^{* n-1}\right)-a^{*}\left(\cdot, t^{n+1}, u_{h}^{* n}\right)\right) \nabla \eta^{n} \cdot \nabla \eta^{n} d \mathbf{x}, \quad 1 \leq n \leq N-1 \\
& \tilde{T}_{4}^{0}=0 .
\end{aligned}
$$

Combining (4.4) and (4.8) we see that

$$
\begin{aligned}
& \sum_{n=0}^{N-1} \Delta t\left\|\delta_{t} \eta^{n}\right\|_{0, \Omega}^{2}+A^{* N}\left(u_{h}^{* N-1}, \eta^{N}, \eta^{N}\right) \\
& \quad=\sum_{n=0}^{N-1} \Delta t\left(\sum_{k=1}^{4} \tilde{T}_{k}^{n}-\left(\delta_{t} \xi^{n}, \delta_{t} \eta^{n}\right)-\left(E^{n, \theta}, \delta_{t} \eta^{n}\right)\right) .
\end{aligned}
$$

We now estimate the right hand side terms of (4.10) and for ease of 
notation adopt again the norm $\||\cdot|\|$, where

$$
\||\phi|\|^{2} \equiv \sum_{n=0}^{N} \Delta t\left\|\phi^{n}\right\|_{0, \Omega}^{2} .
$$

By the Schwartz inequality we have

$$
\sum_{n=0}^{N} \Delta t\left(\delta_{t} \xi^{n}, \delta_{t} \eta^{n}\right) \leq\left\|\left|\delta_{t} \xi\|\| \cdot\left\|\mid \delta_{t} \eta\right\| \|\right.\right.
$$

and

$$
\sum_{n=0}^{N} \Delta t\left(E^{n, \theta}, \delta_{t} \eta^{n}\right) \leq \Delta t\left\|u_{t t}\right\|_{L^{2}\left((0, T) ; L^{2}(\Omega)\right.}\left\|\left|\delta_{t} \eta\right|\right\|
$$

Now using summation by parts we have that

$$
\begin{aligned}
\sum_{n=0}^{N-1} \Delta & t \tilde{T}_{1}^{n}= \\
= & \sum_{n=0}^{N-1} \Delta t \int_{\Omega}\left(a^{*}\left(\mathbf{x}, t^{n+1}, u_{h}^{* n}\right)-a^{*}\left(\mathbf{x}, t^{n+1}, u^{n}\right)\right) \nabla \tilde{u}_{h}^{n+1} \cdot \nabla\left(\delta_{t} \eta^{n}\right) \\
= & \sum_{n=0}^{N-1}\left\{-\int_{\Omega} \Delta t \delta_{t}\left(\left[a^{*}\left(\mathbf{x}, t^{n}, u_{h}^{* n-1}\right)-a^{*}\left(\mathbf{x}, t^{n}, u^{n-1}\right)\right] \nabla \tilde{u}_{h}^{n}\right) \cdot \nabla \eta^{n} d \mathbf{x}\right\} \\
& +\int_{\Omega}\left(a^{*}\left(\mathbf{x}, t^{N}, u_{h}^{* N-1}\right)-a^{*}\left(\mathbf{x}, t^{N}, u^{N-1}\right)\right) \nabla \tilde{u}_{h}^{N} \cdot \nabla \eta^{N} d \mathbf{x} \\
= & \sum_{n=1}^{N-1}-\left[\int_{\Omega} \delta_{t}\left(a^{*}\left(\mathbf{x}, t^{n}, u_{h}^{* n-1}\right)-a^{*}\left(\mathbf{x}, t^{n}, u^{n-1}\right)\right) \nabla \tilde{u}_{h}^{n} \cdot \nabla \eta^{n} d \mathbf{x}\right. \\
& \left.+\int_{\Omega}\left(a^{*}\left(\mathbf{x}, t^{n}, u_{h}^{* n-1}\right)-a^{*}\left(\mathbf{x}, t^{n}, u^{n-1}\right)\right) \nabla \delta_{t} \tilde{u}_{h}^{n} \cdot \nabla \eta^{n} d \mathbf{x}\right] \Delta t \\
& +\int_{\Omega}\left(a^{*}\left(\mathbf{x}, t^{N}, u_{h}^{* N-1}\right)-a^{*}\left(\mathbf{x}, t^{N}, u^{N-1}\right)\right) \nabla \tilde{u}_{h}^{N} \cdot \nabla \eta^{N} d \mathbf{x} .
\end{aligned}
$$

We note that 


$$
\begin{aligned}
\delta_{t}\left(a^{*}\left(\mathbf{x}, t^{n}, u_{h}^{* n-1}\right)-a^{*}\left(\mathbf{x}, t^{n}, u^{n-1}\right)\right) \\
=\delta_{t}\left(\frac{\partial a^{*}}{\partial u}\left(\mathbf{x}, t^{n}, \bar{\nu}^{n-1}\right)\left(u_{h}^{* n-1}-u^{n-1}\right)\right) \\
=\delta_{t}\left(\frac{\partial a^{*}}{\partial u}\left(\mathbf{x}, t^{n}, \bar{\nu}^{n-1}\right)\right)\left(u_{h}^{* n}-u^{n}\right) \\
\quad+\frac{\partial a^{*}}{\partial u}\left(\mathbf{x}, t^{n+1}, \bar{\nu}^{n}\right) \delta_{t}\left(u_{h}^{* n-1}-u^{n-1}\right)
\end{aligned}
$$

for any $\bar{\nu}^{n-1}$ lying between $u_{h}^{* n-1}$ and $u^{n-1}$.

Using the Lipschitz continuity of $a^{*}$ and the Schwarz inequality we deduce that

$$
\begin{aligned}
\sum_{n=0}^{N-1} \Delta & t \tilde{T}_{1}^{n} \\
\leq & \sum_{n=1}^{N-1} \Delta t\left(\left\|\delta_{t} \frac{\partial a}{\partial u}\right\|_{\infty, \Omega}\left\|\nabla \tilde{u}_{h}\right\|_{\infty, \Omega}\left(\left\|\eta^{n}\right\|_{0, \Omega}+\left\|\xi^{n}\right\|_{0, \Omega}\right)\right. \\
& +\left\|\frac{\partial a}{\partial u}\right\|_{\infty}\left\|\nabla \tilde{u}_{h}\right\|_{\infty, \Omega}\left(\left\|\partial_{t} \eta^{n-1}\right\|_{0, \Omega}+\left\|\partial_{t} \xi^{n-1}\right\|_{0, \Omega}\right) \\
& \left.+\left\|\frac{\partial a}{\partial u}\right\|_{\infty}\left\|\nabla \delta_{t} \tilde{u}_{h}^{n}\right\|_{\infty, \Omega}\left(\left\|\eta^{n-1}\right\|_{0, \Omega}+\left\|\xi^{n-1}\right\|_{0, \Omega}\right)\right)\left\|\nabla \eta^{n}\right\|_{0, \Omega} \\
& +\left(\left\|\xi^{N-1}\right\|_{0, \Omega}+\left\|\eta^{N-1}\right\|_{0, \Omega}\right)\left\|\nabla \tilde{u}_{h}^{N}\right\|\left\|_{\infty, \Omega}\right\| \nabla \eta^{N} \|_{0, \Omega} \\
\leq & C_{1}\left(\||\eta\|+\| \xi|\|+\left\|\delta_{t} \eta\right\|+\left\|\delta_{t} \xi \mid\right\|\right) \cdot\|\nabla \eta\| \\
& +C_{1}\left(\left\|\xi^{N-1}\right\|_{0, \Omega}+\left\|\eta^{N-1}\right\|_{0, \Omega}\right)\left\|\nabla \eta^{N}\right\|_{0, \Omega}
\end{aligned}
$$

where $C_{1}$ depends on $\left\|\frac{\partial^{2} a}{\partial t \partial u}\right\|_{\infty},\left\|\nabla \tilde{u}_{h}\right\|_{\infty},\left\|\frac{\partial a}{\partial u}\right\|$, and $\left\|\nabla \delta_{t} \tilde{u}_{h}^{n}\right\|$. 
We have

$$
\begin{aligned}
& \sum_{n=0}^{N-1} \Delta t \tilde{T}_{2}^{n}= \\
& =\sum_{n=0}^{N-1} \Delta t \int_{\Omega}\left(a^{*}\left(\mathbf{x}, t^{n+1}, u^{n}\right)-a\left(\mathbf{x}, t^{n+1}, u^{n+1}\right)\right) \nabla \tilde{u}_{h}^{n+1} \cdot \nabla \partial_{t} \eta^{n} \\
& =\sum_{n=0}^{N-1} \Delta t\left(\int_{\Omega}\left(a^{*}\left(\mathbf{x}, t^{n+1}, u^{n}\right)-a^{*}\left(\mathbf{x}, t^{n+1} u^{n+1}\right)\right) \nabla \tilde{u}_{h}^{n+1} \cdot \nabla \partial_{t} \eta^{n}\right. \\
& \left.\quad+\int_{\Omega}\left(a^{*}\left(\mathbf{x}, t^{n+1}, u^{n+1}\right)-a\left(\mathbf{x}, t^{n+1}, u^{n+1}\right)\right) \nabla \tilde{u}_{h}^{n+1} \cdot \nabla \partial_{t} \eta^{n}\right) \cdot(
\end{aligned}
$$

Again we apply summation by parts which yields

$$
\begin{aligned}
& \sum_{n=0}^{N-1} \Delta t \tilde{T}_{2}^{n}= \\
& =\sum_{n=1}^{N-1}-(\Delta t)^{2}\left(\int_{\Omega} \delta_{t}\left(\frac{\partial a^{*}}{\partial u}\left(\cdot, t^{n}, \theta^{n-1}\right) \delta_{t} u^{n-1} \nabla \tilde{u}_{h}^{n}\right) \cdot \nabla \eta^{n} d \mathbf{x}\right. \\
& \quad-\Delta t \int_{\Omega} \frac{\partial a^{*}}{\partial u}\left(\cdot, t^{N}, \theta^{N-1}\right) \delta_{t} u^{N-1} \nabla \tilde{u}_{h}^{N} \cdot \nabla \eta^{N} d \mathbf{x} \\
& \quad-\sum_{n=1}^{N-1} \Delta t \int_{\Omega} \delta_{t}\left(\left(a^{*}-a\right)^{n} \nabla \tilde{u}_{h}^{n}\right) \cdot \nabla \eta^{n} d \mathbf{x} \\
& \quad+\int_{\Omega}\left(a^{*}-a\right)\left(\cdot, t^{N} u^{N}\right) \nabla \tilde{u}_{h}^{N} \cdot \nabla \eta^{N} d \mathbf{x} .
\end{aligned}
$$

Thus, from (4.17) 


$$
\begin{aligned}
& \sum_{n=0}^{N-1} \Delta t \tilde{T}_{2}^{n} \leq \\
& C\left(\Delta t\left\|\frac{\partial a^{*}}{\partial u}\right\|_{\infty}\left\|\nabla \tilde{u}_{h}\right\|_{\infty, \Omega}\left\|\frac{\partial u^{2}}{\partial t^{2}}\right\|_{L^{2}\left((0, T) ; L^{2}(\Omega)\right)}\right. \\
& +\Delta t\left\|\frac{\partial^{2} a^{*}}{\partial t \partial u}\right\|_{\infty} \cdot\left\|\frac{\partial u}{\partial t}\right\|_{L^{2}\left((0, T) ; L^{2}(\Omega)\right)} \cdot\left\|\nabla \tilde{u}_{h}\right\|_{\infty, \Omega} \\
& \left.+\Delta t\left\|\frac{\partial a^{*}}{\partial u}\right\|_{\infty}\left\|\delta_{t} u^{n-1}\right\|_{L^{2}\left((0, T), L^{2}(\Omega)\right)} \cdot\left\|\nabla\left(\tilde{u}_{h}\right)_{t}\right\|_{\infty, \Omega}\right)\|\mid \nabla \eta\| \\
& +\Delta t\left\|\frac{\partial a^{*}}{\partial u}\right\|_{\infty}\left\|\frac{\partial u^{N-1}}{\partial t}\right\|_{0, \Omega} \cdot\left\|\nabla \eta^{N}\right\|\left\|\nabla \tilde{u}_{h}\right\|_{\infty} \\
& +\left\|\frac{\partial}{\partial t}\left(a^{*}-a\right)\right\|_{L^{2}\left((0, T) ; L^{2}(\Omega)\right)} \sum_{\ell=0}^{1}\left\|\frac{\partial^{\ell} \nabla \tilde{u}_{h}}{\partial t^{\ell}}\right\|_{\infty} \cdot|\|\nabla \eta\|| \\
& +\left\|a^{*}-a\right\|_{L^{\infty}\left((0, T) ; L^{2}(\Omega)\right)}\left\|\nabla \tilde{u}_{h}\right\|_{\infty} \cdot\left\|\nabla \eta^{N}\right\| .
\end{aligned}
$$

We have

$$
\begin{aligned}
& \sum_{n=0}^{N-1} \Delta t \tilde{T}_{3}^{n} \leq \\
& \quad \leq\|\| f-f^{*}\|\|\|\| \delta_{t} \eta \| \mid \\
& \quad \leq C\left(\| \| \eta \left\|\left|+\|\mid\| \xi\left\|+h^{q+p-1}\right\| u \|_{L^{2}\left((0, T), H^{q}(\Omega)\right)}\right) \cdot\left|\left\|\delta_{t} \eta\right\|\right|\right.\right.
\end{aligned}
$$


In addition we see that

$$
\begin{aligned}
& \sum_{n=0}^{N-1} \Delta t \tilde{T}_{\mathbf{4}}^{n} \leq \\
& \quad \leq \sum_{n=0}^{N-1} \Delta t\left(\left\|\delta_{t} \eta^{n}\right\|_{0, \Omega}+\left\|\frac{\delta \xi^{n}}{\partial t}\right\|_{0, \Omega}\right) h^{-2}\left\|\eta^{n}\right\|_{0, \Omega}\left\|\nabla \eta^{n}\right\|_{0, \Omega} .
\end{aligned}
$$

Applying results from Theorem 3.1 for $q=2$ and $p=1$ we have that

$$
\left\|\eta^{n}\right\|_{0, \Omega} \leq C\left(h^{2}+\Delta t\right)
$$

Letting $\Delta t=O\left(h^{2}\right)$ we have that

$$
\begin{aligned}
& \sum_{n=0}^{N-1} \Delta t \tilde{T}_{4}^{n} \leq \\
& \leq \quad\left(\left|\left\|\delta_{t} \eta\right\|\right|+\left\|\frac{\partial \xi}{\partial t}\right\|_{L^{2}\left((0, T) ; L^{2}(\Omega)\right)}\right. \\
& \left.\quad+h^{2}\left\|\frac{\partial u}{\partial t}\right\|_{\left.L^{2}(0, T), H^{2}(\Omega)\right)}\right)\||\nabla \eta|\| .
\end{aligned}
$$

Combining the estimates (4.15), (4.18), (4.19) and (4.21) we conclude that 


$$
\begin{aligned}
\|\| \delta_{t} \eta \| \mid & +A^{* N}\left(u_{h}^{* N-1}, \eta^{N}, \eta^{N}\right) \\
\leq & \left|\left\|\delta_{t} \xi\right\|\right|\left\|\delta_{t} \eta\right\|\left|+\Delta t\left\|u_{t t}\right\|_{L^{2}\left((0, T) ; L^{2}(\Omega)\right)}\right|\left\|\delta_{t} \eta\right\| \mid \\
& +C_{1}\left(|\|\eta\|+|\|\xi\|\left|+\left\|\delta_{t} \eta\right\|+\right|\left\|\delta_{t} \xi\right\| \mid\right)|\|\nabla \eta\|| \\
& +C_{1}\left(\left\|\xi^{N-1}\right\|_{0, \Omega}+\left\|\eta^{N-1}\right\|_{0, \Omega}\right)\left\|\nabla \eta^{N}\right\|_{0, \Omega} \\
& +C_{2} \Delta t\|u\|_{H^{2}\left((0, T) ; L^{2}(\Omega)\right)}\|\eta \eta\| \\
& +C_{2}\left(|\|\eta\||+|\|\xi\||+h^{2}\|u\|_{L^{2}\left((0, T) ; H^{2}(\Omega)\right)}\right) \\
& +\left|\left\|\delta_{t} \eta\right\|\right|+\| \frac{\partial \xi \|_{\partial t}}{} \\
& +h^{2}\left\|\frac{\partial u}{\partial t}\right\|_{L^{2}\left((0, T) ; H^{2}(\Omega)\right)}\|\nabla \eta\| L_{\left.L^{2}(\Omega)\right)}
\end{aligned}
$$

where $C_{1}$ and $C_{2}$ depend on $\left\|\frac{\partial^{2} a^{*}}{\partial t \partial u}\right\|_{\infty, \Omega},\left\|\frac{\partial a^{*}}{\partial u}\right\|_{\infty, \Omega}$ and $\left\|\nabla \tilde{u}_{k}\right\|_{\infty, \Omega} \cdot C_{2}$ also depends on $\left\|\nabla \tilde{u}_{h_{t}}\right\|_{\infty}$.

Using approximation theory and nonlinear results from Theorem 3.1 and Lemma 3.1, we have

$$
\left\|\eta^{N}\right\|+|\|\nabla \eta\|| \leq C\left(h^{2}+\Delta t\right)
$$

Thus for $\Delta t=O\left(h^{2}\right)$ we have 


$$
\begin{aligned}
& \left|\left\|\delta_{t} \eta\right\|\right|+A^{*}\left(\tilde{u}_{h}^{* N-1}, \eta^{N}, \eta^{N}\right) \\
& \quad \leq \epsilon\left|\left\|\delta_{t} \eta\right\|\right|^{2}+C(\epsilon)\left[h^{4}+(\Delta t)^{2}+\epsilon\left\|\nabla \eta^{N}\right\|^{2}\right]
\end{aligned}
$$

which implies that

$$
\left\|\eta^{N}\right\|_{1, \infty, \Omega} \leq C\left(h^{2}+\Delta t\right)
$$

This provides the $L_{\infty}$-time estimate for the backward difference procedure. As stated earlier, the generalization to the case $0 \leq \theta \leq \frac{1}{2}$ follows similar arguments. We finally state these results as a theorem.

Theorem 4.1 Let $u(\mathbf{x}, t)$ be the solution of problem (3.4) and $u_{h}^{* n}(\mathbf{x})$ be the solution of (3.8) with $\theta=0$, where * denotes numerical integration using either interpolation of coefficients or Gauss quadrature. It is assumed that $q=2$ and $p=1$ in (1.12). Then

$$
\left\|\nabla u\left(\cdot, t^{n}\right)-\nabla u_{h}^{* R_{n}}\right\|_{0, \Omega} \leq C\left(h^{2}+\Delta t^{\beta}\right)
$$

for any $n \leq N$ where $\beta=1$ if $0 \leq \theta<\frac{1}{2}$ and $\beta=2$ if $\theta=\frac{1}{2}$. 